\title{
A SPECTROSCOPIC SEARCH FOR CHEMICALLY STRATIFIED WHITE DWARFS IN THE SLOAN DIGITAL
} SKY SURVEY

\author{
P. M. Manseau ${ }^{1}$, P. Bergeron ${ }^{1}$, and E. M. Green ${ }^{2}$ \\ ${ }^{1}$ Département de Physique, Université de Montréal, C.P. 6128, Succ. Centre-Ville, Montréal, Québec H3C 3J7, \\ Canada; manseau@astro.umontreal.ca, bergeron@ astro.umontreal.ca \\ ${ }^{2}$ Steward Observatory, University of Arizona, 933 North Cherry Avenue, Tucson, AZ 85721, USA; bgreen@as.arizona.edu \\ Received 2016 August 20; revised 2016 September 18; accepted 2016 September 19; published 2016 December 13
}

\begin{abstract}
We present a detailed search and analysis of chemically stratified hybrid (traces of helium and hydrogen) white dwarfs in the Sloan Digital Sky Survey (SDSS). Only one stratified white dwarf, PG 1305-017, was known prior to this analysis. The main objective is to confirm the existence of several new stratified objects. We first describe our new generation of stratified model atmospheres, where a thin hydrogen layer floats in diffusive equilibrium on top of a more massive helium layer. We then present the results of our search for hot $\left(T_{\text {eff }}>30,000 \mathrm{~K}\right)$ white dwarfs with a hybrid spectral type among the $\sim 38,000$ white dwarf spectra listed in the SDSS. A total of 51 spectra were retained in our final sample, which we analyze using spectroscopic fits to both chemically homogeneous and stratified model atmospheres. We identify 14 new stratified white dwarfs in the SDSS sample. From these results, we draw several conclusions regarding the physical processes that might explain the presence of helium in the atmospheres of all the stars in our sample.
\end{abstract}

Key words: stars: abundances - stars: atmospheres - stars: evolution - stars: fundamental parameters - white dwarfs

Supporting material: extended figures

\section{INTRODUCTION}

White dwarf stars are stellar remnants which, having exhausted the nuclear fuel in their core, simply cool off over billions of years as degenerate objects with almost constant radii. As such, white dwarfs are often erroneously viewed as simple objects-if not simplistic-even in our scientific community. However, a careful reading of the cooling theory of white dwarfs, such as that presented in Fontaine et al. (2001), clearly shows that the evolution of these degenerate stars is far from being uneventful. The physics of their interiors requires state-of-the-art calculations, involving almost all branches of physics, in order to provide realistic and quantitative predictions about their evolution as a function of time. Hence, white dwarfs are, in fact, quite complex objects.

A most difficult puzzle, which still remains to be solved in detail, is the evolution of the surface composition as a function of cooling age-or what is referred to as the spectral evolution of white dwarfs. In the mid-eighties, the study of the spectral evolution of white dwarfs was one of the most active fields of research in the white dwarf community (see Fontaine \& Wesemael 1987 for an excellent review). Thanks to large colorimetric and spectroscopic surveys, such as the PalomarGreen (PG) Survey (Green et al. 1986), that significantly increased the number of known white dwarfs-in particular, at the bright end of the luminosity function-some clear patterns of the variation of spectral types as a function of temperature started to emerge for the first time.

Fontaine \& Wesemael (1987) summarized, in their Figures 1 and 2, the trace element patterns identified at the time, along with the various physical mechanisms that could compete with gravitational settling to alter the chemical composition of the outer layers of white dwarfs, as they evolve along the cooling sequence. Such physical mechanisms include ordinary diffusion, convective mixing, convective dredge-up from the core, accretion from the interstellar medium (or circumstellar material), radiative acceleration, and stellar winds. One of the most striking features is a temperature range, between $T_{\text {eff }} \sim 45,000$ and 30,000 K, where all white dwarfs are found to have hydrogen-dominated (DA) atmospheres-the so-called DB gap (see Figure 3 of Fontaine \& Wesemael 1987). However, non-DA stars are found in large numbers above and below this gap (the DO and DB stars, respectively).

Fontaine \& Wesemael (1987) were among the first to propose a consistent model for the spectral evolution of white dwarfs that could account for the existence of this DB gap. In their model, a significant fraction of all white dwarfs evolved from helium-rich PG 1159 stars. As these stars cool down, residual hydrogen-thoroughly mixed in the helium-rich envelope - eventually diffuses upward, and a thicker hydrogen atmosphere is gradually built up at the surface, transforming a helium-rich atmosphere into a hydrogen-rich atmosphere. The constraint that all white dwarfs below the blue edge of the DB gap at $\sim 45,000 \mathrm{~K}$ have hydrogen-rich atmospheres thus imposes a lower limit to the total amount of hydrogen left in the envelope of post-AGB progenitors. At lower effective temperatures, near the red edge of the DB gap, DA stars can be transformed into helium-atmosphere DB white dwarfs, as a result of the convective dilution of their thin radiative hydrogen layer $\left(M_{\mathrm{H}} \sim 10^{-15} M_{\odot}\right)$ with the deeper convective helium envelope (see MacDonald \& Vennes 1991 for a full quantitative exploration of this convective mixing scenario).

In the context of this float-up model, DAO stars have been interpreted as intermediate objects undergoing a spectroscopic metamorphosis. If this interpretation is correct, the helium abundance distribution in such objects is given by the diffusive equilibrium abundance profile, and the atmospheres of DAO stars should consequently have chemically stratified atmospheres. Vennes \& Fontaine (1992) even demonstrated, from theoretical considerations, that stratified configurations are the 
only viable explanation for the presence of helium in DAO stars - with the possible exception of stellar winds. Bergeron et al. (1994, see also Gianninas et al. 2010) explored this idea more quantitatively by analyzing a large sample of bright DAO stars with homogeneous and stratified models, and found that most DAO stars were better interpreted in terms of chemically homogeneous compositions, resulting, most likely, from the presence of a weak stellar wind above $T_{\text {eff }} \sim 60,000 \mathrm{~K}$. One glaring exception to this general trend is PG 1305-017, whose optical spectrum was better reproduced with chemically stratified models (see Figure 8 of Bergeron et al.). With an effective temperature of $T_{\text {eff }} \sim 45,000 \mathrm{~K}$, PG 1305-017 lies at the blue edge of the DB gap and fits perfectly within the picture of the float-up model discussed above.

The Sloan Digital Sky Survey (SDSS) is certainly among the most important developments in the last decade, in terms of observational data for white dwarf stars, since the PG survey (see, e.g., Eisenstein et al. 2006a). The SDSS observed $10,000 \mathrm{deg}^{2}$ of high-latitude sky in five bandpasses (ugriz) and produced images in these five bandpasses from which galaxies, quasars, and stars were selected for follow-up spectroscopy. Not only has the SDSS increased the number of spectroscopically confirmed white dwarfs by more than an order of magnitude since the last published version of the McCook \& Sion catalog (McCook \& Sion 1999), it has provided a phenomenal source of homogeneous photometric observations in the ugriz system, as well as optical spectroscopy for many objects. However, the selection effects in this survey are important, as discussed in Kleinman et al. (2004) and Eisenstein et al. (2006a), and therefore, it cannot be considered as a complete survey in any sense.

One of the most significant results from the SDSS has been the discovery of extremely hot $\left(T_{\text {eff }} \gtrsim 30,000 \mathrm{~K}\right)$ DB stars that partially fill the DB gap (Eisenstein et al. 2006b). The number of such hot DB stars remains low, however, compared to cooler siblings_-about a factor of 2.5 lower-and thus, we are dealing with a deficiency of DB stars in this range of temperature rather than a true gap. Bergeron et al. (2011) presented a detailed analysis of 108 relatively bright DB white dwarfs based on model atmosphere fits to high signal-to-noise optical spectroscopy. They concluded from their analysis that a small fraction of white dwarf stars are probably born as hydrogen-deficient stars, with negligible amounts of hydrogen in their envelope, that remain hydrogen-deficient throughout their subsequent evolution. The existence of extremely hot DB stars in the DB gap by Eisenstein et al. certainly supports this conclusion. Bergeron et al. also concluded from statistical arguments that only a single DB white dwarf could have been expected in the PG survey, and therefore, it was not surprising that none had been found.

Despite the fact that the SDSS has significantly increased the number of spectroscopically identified white dwarfs, its potential in terms of the study of the spectral evolution of white dwarfs has never been fully exploited. In particular, there has not been any systematic search for chemically stratified DAO or DAB white dwarfs that could further validate the floatup scenario. In addition, the identification of a large number of such hybrid stratified white dwarfs could shed some light on the range of hydrogen layer mass left during the post-AGB phase. We present in this paper the results of such an investigation. The model atmospheres with homogeneous and stratified compositions used in this analysis are first described in Section 2. Our search of the SDSS spectroscopic database is then discussed in Section 3, along with independent spectra that were acquired for the purpose of this project. Our spectroscopic analysis and interpretation follow in Sections 4 and 5, respectively.

\section{THEORETICAL FRAMEWORK}

\subsection{Model Atmospheres and Synthetic Spectra}

Our model atmospheres and synthetic spectra are derived from the LTE model atmosphere code originally described in Bergeron et al. (1995, 2011), and references therein, with several improvements discussed in Tremblay \& Bergeron (2009); in particular, the improved calculations for the Stark broadening of hydrogen lines, which include nonideal perturbations from protons and electrons directly inside the line profile calculations. With respect to the analysis of DAO stars presented in Bergeron et al. (1994), we now systematically include all the absorption lines from ionized helium, in the UV in particular, that were treated only partially in our earlier calculations.

The atmospheric parameters of our chemically homogeneous models cover a range of $T_{\text {eff }}=30,000$ (1000) 60,000 K (where the quantity in parentheses indicates the step size), log $g=7.0(0.5) 8.5$, and a wide range of helium abundances of $\log y \equiv \log N(\mathrm{He}) / N(\mathrm{H})=-5.0$ (1.0) 5.0 covering all cases where both hydrogen and helium are considered as trace elements. The upper limit of our grid is set at $T_{\text {eff }}=60,000 \mathrm{~K}$ because white dwarfs above this temperature are expected to have homogeneous chemical compositions, due to the presence of a weak stellar wind (see Gianninas et al. 2010 and our discussion below). Pure hydrogen and pure helium models were calculated as well, for illustrative purposes (see below).

Our model atmospheres with stratified chemical compositions have been calculated using the same approach as that discussed in Bergeron et al. (1994), which is based on the formalism described at length in Jordan \& Koester (1986) and Vennes \& Fontaine (1992). These models are defined in terms of the total amount of hydrogen floating in diffusive equilibrium on top of the helium envelope, $q_{\mathrm{H}} \equiv M_{\mathrm{H}} / M_{\star}$, where $M_{\mathrm{H}}$ and $M_{\star}$ represent, respectively, the total mass of the hydrogen layer and the mass of the star. The equilibrium abundance profile, $N(\mathrm{H}) / N(\mathrm{He})$, is obtained by solving an equation similar to Equation (1) of Vennes \& Fontaine (1992), and thus becomes a function of optical depth. Examples of such equilibrium profiles (expressed here as $N_{\mathrm{H}} / N_{\text {total }}$ ) are displayed in Figure 1 for a model with $T_{\text {eff }}=45,000 \mathrm{~K}$ (i.e., at the temperature of PG 1305-017), $\log g=8$, and for various values of the thickness of the hydrogen layer. As the value of $q_{\mathrm{H}}$ increases, the transition region between the pure hydrogen and the pure helium layers occurs increasingly deeper in the star. Because the line forming region lies near $\tau_{R} \sim 0.1-1$, we expect white dwarfs in this temperature range to show hybrid spectra for $\log q_{\mathrm{H}} \sim-17$ to -16 . Otherwise, if the hydrogen is too thin or thick, the star will appear as a normal DB or DA star, respectively. Because atomic diffusion can only operate in the absence of convective energy transport, we assume that our stratified models are purely radiative - an hypothesis that must be verified a posteriori (see below).

As discussed below, the surface gravity significantly affects the relative strength of hydrogen and helium lines for a given value of $q_{\mathrm{H}}$. As such, the range of hydrogen layer masses must 


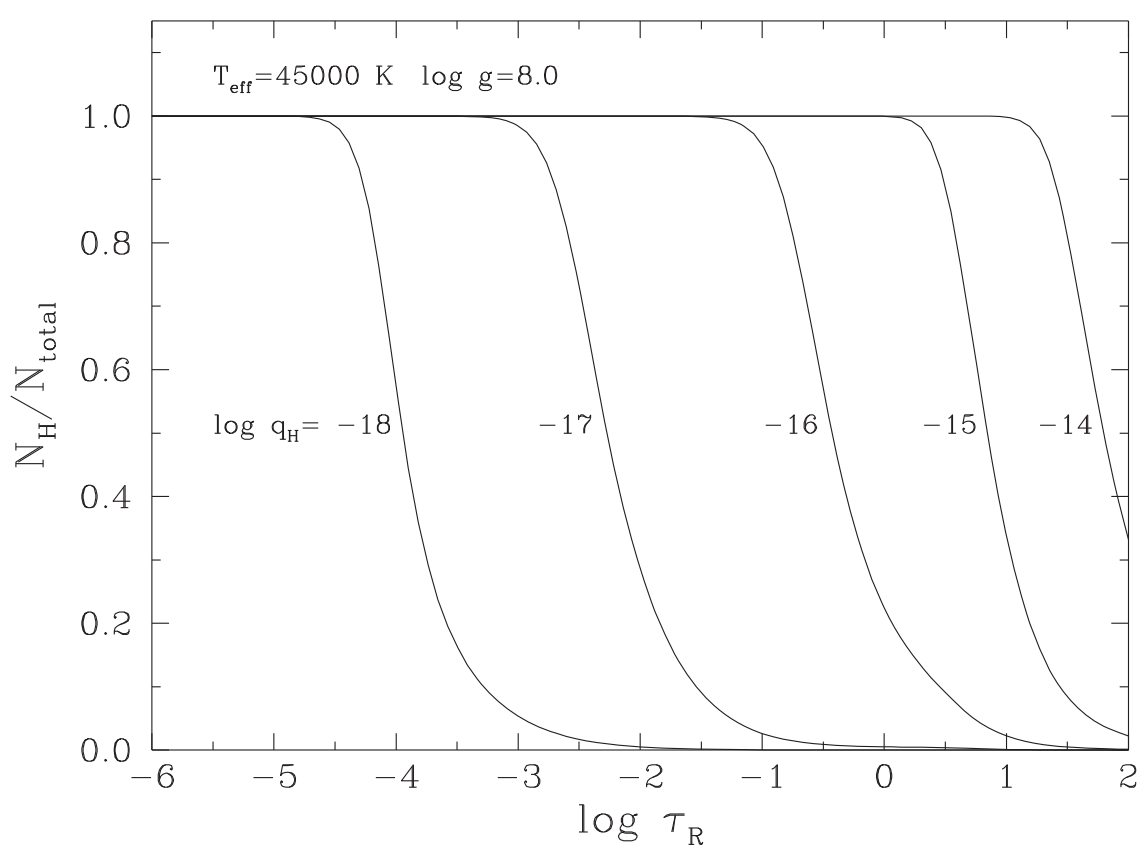

Figure 1. Hydrogen abundance profile, $N_{\mathrm{H}} / N_{\text {total }}$ as a function of Rosseland optical depth, in a model with $T_{\text {eff }}=45,000 \mathrm{~K}$, $\log g=8$, and for various values of the hydrogen fractional mass $q_{\mathrm{H}}$.

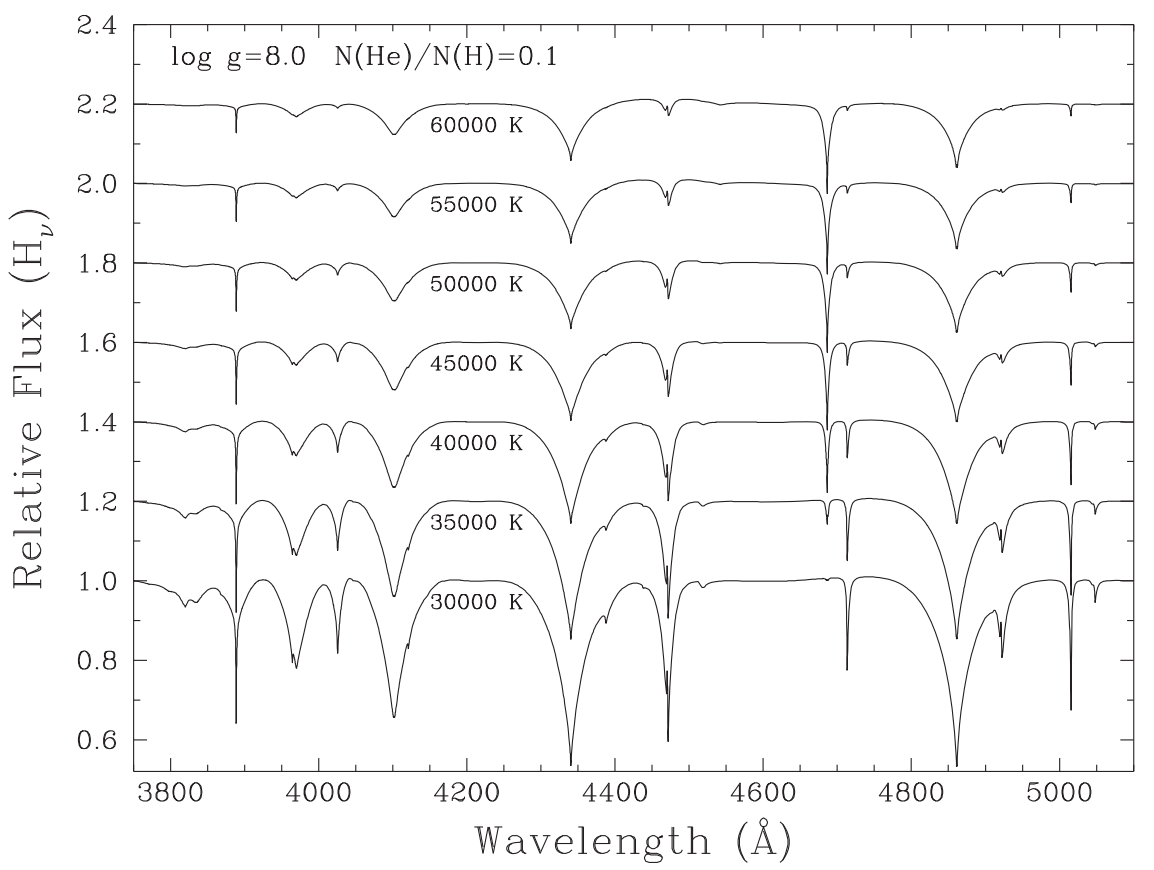

Figure 2. Synthetic spectra for models with homogeneous chemical compositions at $\log g=8, N(\mathrm{He}) / N(\mathrm{H})=0.1$, and for various effective temperatures.

be adjusted as a function of $\log g$. For instance, we use a range of $\log q_{\mathrm{H}}=-16.50(0.50)-12.50$ at $\log g=7.0$, and $\log q_{\mathrm{H}}=-18.75(0.50)-14.75$ at $\log g=8.5$. In the context of stratified atmospheres, it is useful to define a quantity $Q \equiv 3 \log g+2 \log q_{\mathrm{H}}$, which allows spectra for a given value of $Q$ to look relatively similar (with respect to the strength of the helium lines) in the $\log g-\log q_{\mathrm{H}}$ plane. Models calculated with $Q=-12.0(1.0)-4.0$, and having the same range of $T_{\text {eff }}$ and $\log g$ as the homogeneous grid, embrace the entire spectral properties of the white dwarfs identified in the SDSS.

\subsection{Exploration of the Parameter Space}

For completeness, we present in this section the results of our synthetic spectrum calculations (see also Vennes \& Fontaine 1992), which will also serve as a guide for our search of hybrid spectra in the SDSS spectroscopic database.

Illustrative spectra at $\log g=8.0$ for homogeneous $-N(\mathrm{He}) / N(\mathrm{H})=0.1$-and stratified- $\log q_{\mathrm{H}}=-16.5-$ compositions are displayed in Figures 2 and 3, respectively, for various effective temperatures. With such a small hydrogen layer mass in the stratified models, the photosphere is always 


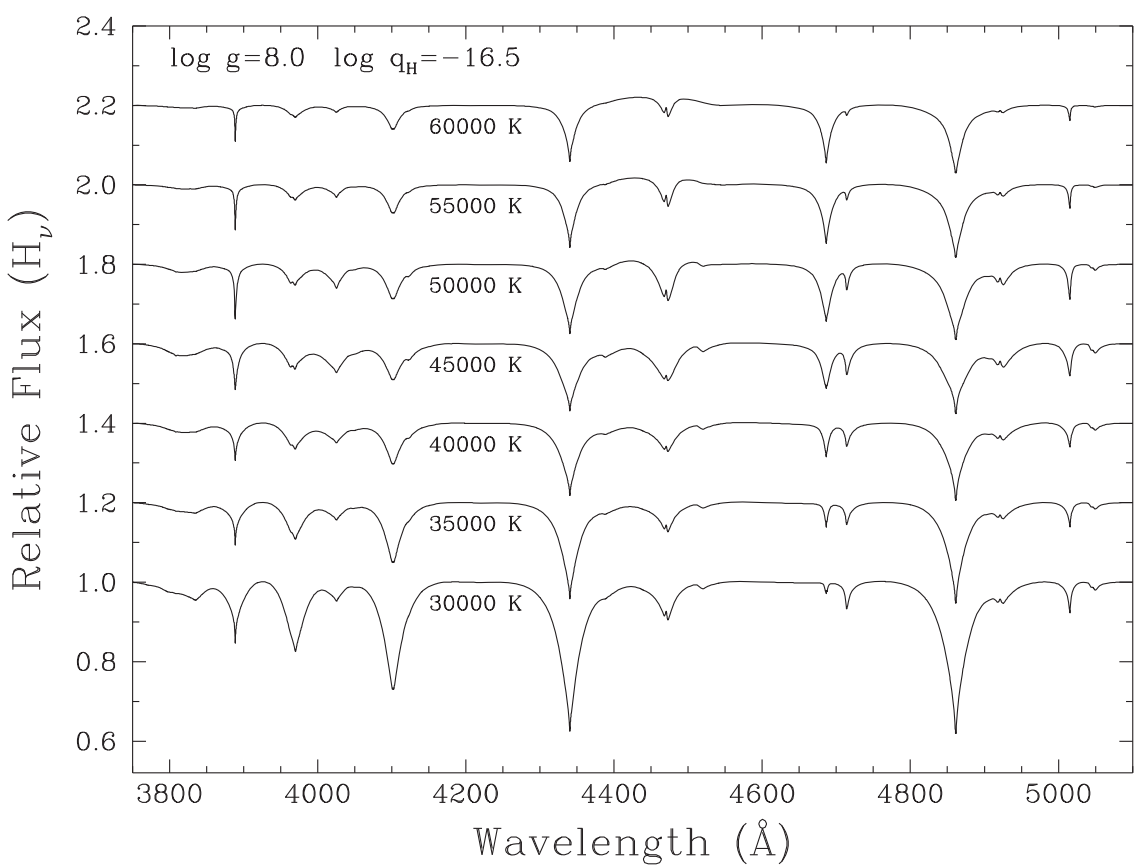

Figure 3. Synthetic spectra for models with stratified chemical compositions at $\log g=8, \log q_{\mathrm{H}}=-16.5$, and for various effective temperatures.

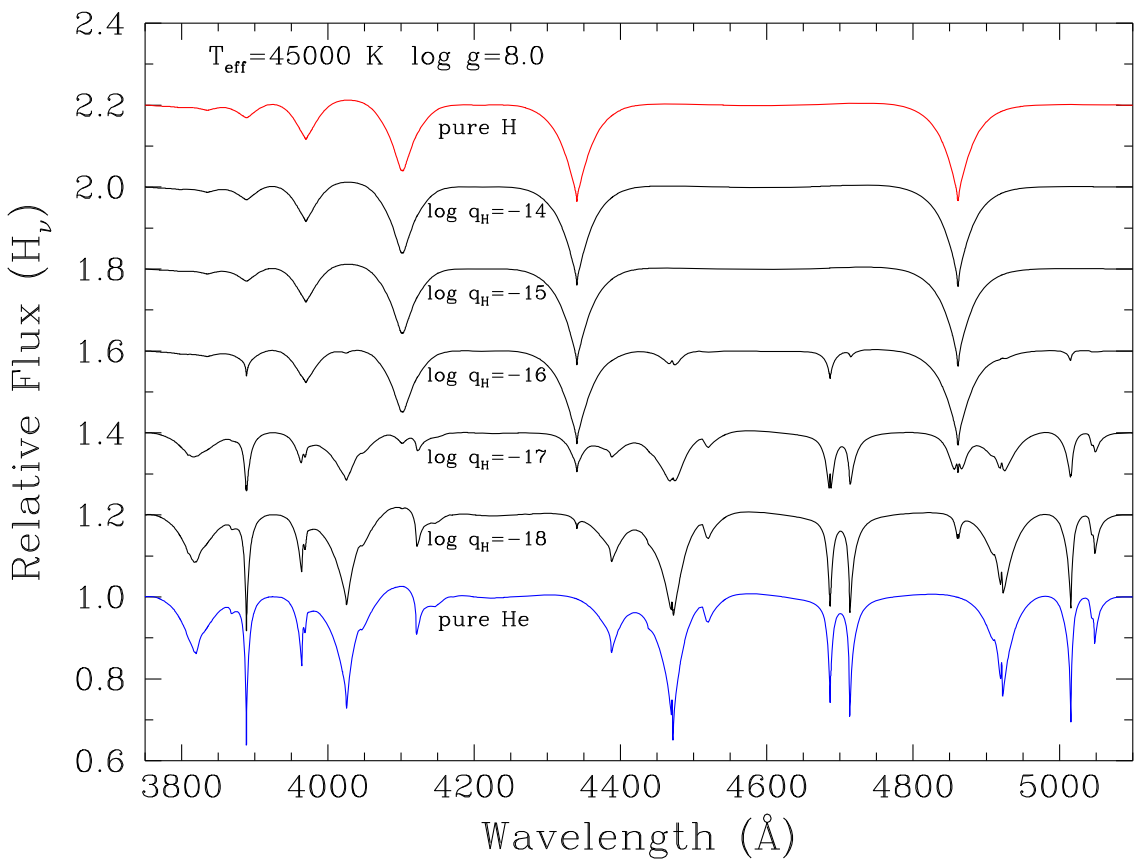

Figure 4. Synthetic spectra for models with stratified chemical compositions at $T_{\text {eff }}=45,000 \mathrm{~K}, \log g=8$, and for various masses of the hydrogen layer $\log q_{\mathrm{H}}$. For comparison, pure hydrogen and pure helium spectra are shown at the top and at the bottom, respectively.

polluted with enough helium from the deeper envelope that helium lines are visible at all temperatures, particularly the He I $\lambda 4471$ absorption feature. In both sets of models, the relative strength of He I $\lambda 4471$ and He II $\lambda 4686$ changes significantly as a function of effective temperature; however, these lines appear sharper and deeper in the homogeneous models, a behavior also observed for other helium lines. This will serve as an important diagnostic to distinguish between chemically homogeneous and stratified white dwarfs.

Illustrative spectra of stratified models at $T_{\mathrm{eff}}=45,000 \mathrm{~K}$, $\log g=8.0$ are displayed in Figure 4 for various values of the hydrogen layer masses; these can be matched with the abundance profiles shown in Figure 1. Also shown for comparison are pure hydrogen and pure helium spectra at the same values of $T_{\text {eff }}$ and $\log g$. The gradual transformation from a pure helium to a pure hydrogen spectrum is clearly observed when the thickness of the hydrogen layer is gradually increased. We can also note that both hydrogen and helium lines become particularly weak for a value of $\log q_{\mathrm{H}}=-17$, when these lines have approximately equal strengths. Also, even when the hydrogen layer is as thin as $\log q_{\mathrm{H}}=-18$ and all the hydrogen lies mostly above the photosphere (see Figure 1), we can still detect hydrogen spectroscopically. 


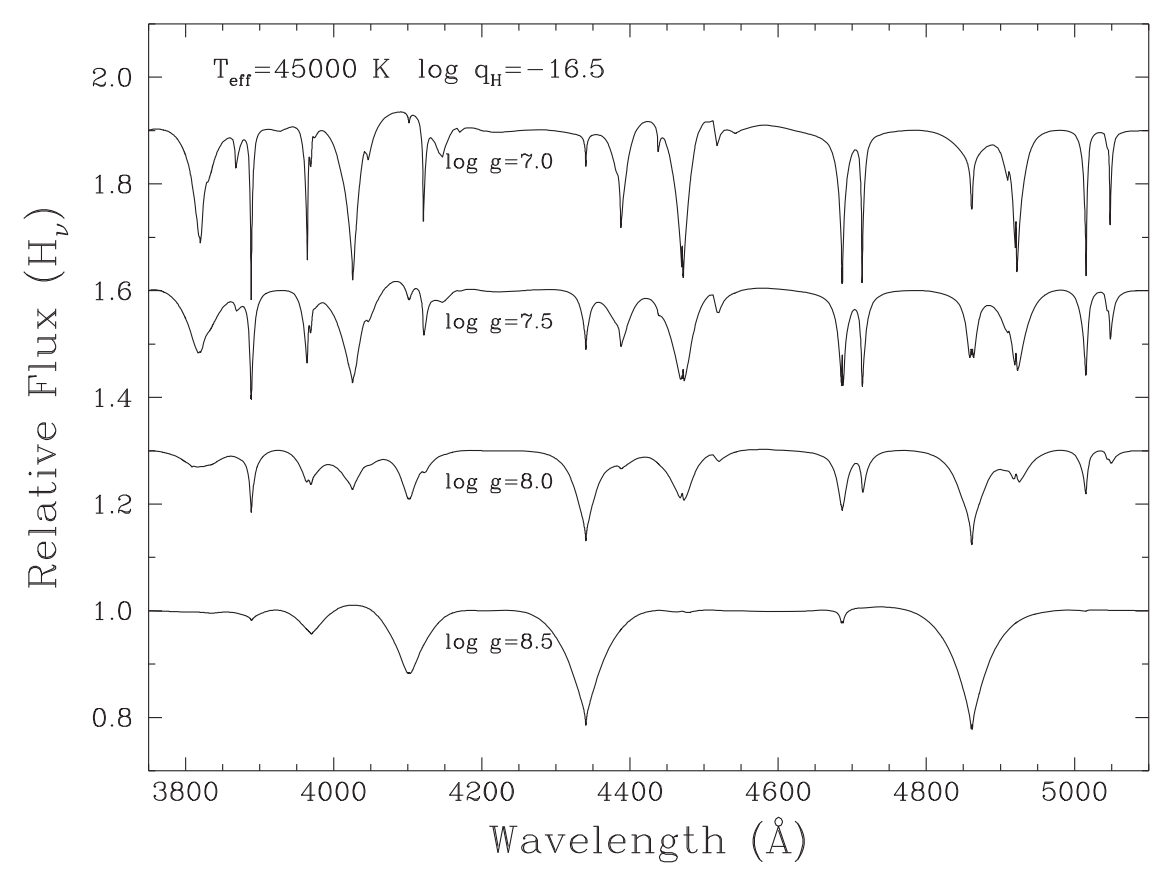

Figure 5. Synthetic spectra for models with stratified chemical compositions at $T_{\text {eff }}=45,000 \mathrm{~K}, \log q_{\mathrm{H}}=-16.5$, and for various values of $\log g$.

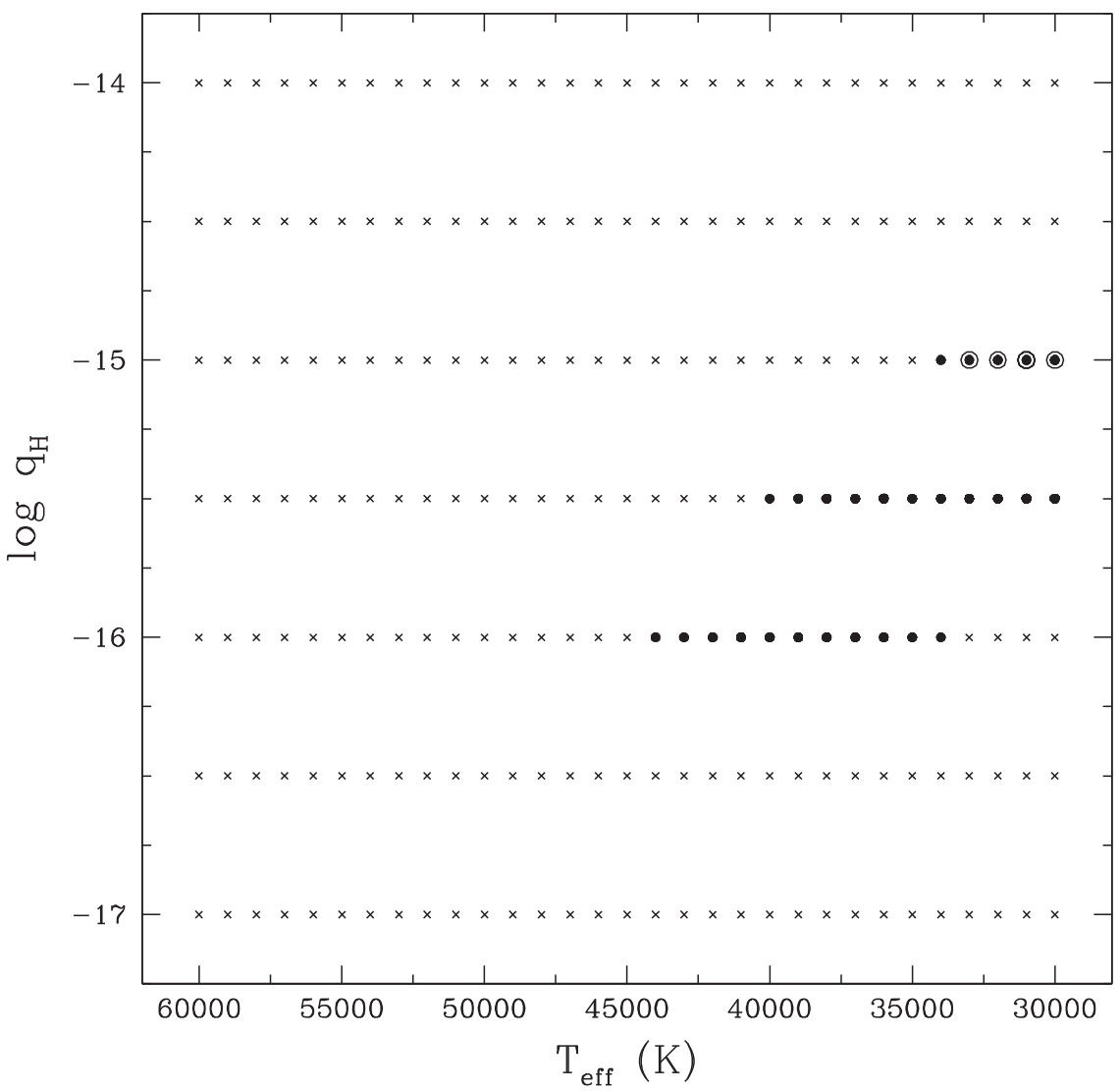

Figure 6. Stratified models in our pure radiative grid at $\log g=8$ that develop a convection zone at the photosphere when the Schwarzschild criterion is being applied. The cross symbols show models that remain purely radiative, whereas the dots correspond to models developing a convection zone above, or within, the $\mathrm{H} / \mathrm{He}$ transition zone. The large circles indicate models where convection is present both above and below the transition zone.

As briefly discussed above, the surface gravity significantly affects the relative strength of hydrogen and helium lines for a given value of $q_{\mathrm{H}}$. This is illustrated in Figure 5, where we show spectra for stratified models at $T_{\mathrm{eff}}=45,000 \mathrm{~K}$, $\log q_{\mathrm{H}}=-16.5$, and for various $\log g$ values. The effect of varying $\log g$ on the abundance profile (not shown here) is similar to that observed in Figure 1, where the transition zone occurs increasingly deeper as $\log g$ increases, resulting in predicted spectra that are eventually completely dominated by hydrogen. Because of this particular behavior, the range of 
hydrogen layer masses in our model grid needs to be adjusted as a function of $\log g$, which led to the introduction of the fitting parameter $Q \equiv 3 \log g+2 \log q_{\mathrm{H}}$ mentioned above.

Because the presence of convective energy transport in the photospheric layers, and in particular in the $\mathrm{H} / \mathrm{He}$ transition region, may result in a complete homogenization of the chemical structure, we need to consider the presence of convection zones in our stratified model atmospheres, which, as mentioned above, are assumed to be purely radiative (see also Section 2.4 of Vennes \& Fontaine 1992). To do so, we applied to our radiative models the Schwarzschild criterion and calculated the resulting convective flux within the standard mixing-length theory, using the so-called $\operatorname{ML} 2 / \alpha=0.8$ parameterization. We show, in Figure 6, the models in our grid at $\log g=8$ that develop a significant convection zone ${ }^{3}$ in the transition region, which could potentially impede the effect of chemical stratification. We see that convection may start to play a role for $T_{\text {eff }} \lesssim 45,000 \mathrm{~K}$, but only for a narrow range of hydrogen layer masses. These models should, thus, be considered uncertain, and we will keep these results in mind for our analysis of spectroscopic data presented below. We also note that our results, displayed in Figure 6, compare favorably with those shown in Figure 2 of Vennes \& Fontaine (1992), with the exception that convection is present in their models at much higher temperatures. This is most likely due to the He II convection zone, which we believe is too weak in our models to homogenize the chemical structure (see below). Finally, we point out that more-detailed 3D hydrodynamical model atmospheres have recently become available for hydrogen-rich white dwarfs (Tremblay et al. 2013, 2015, and references therein), although such calculations are still not available for DB stars. Furthermore, for these calculations to be relevant in the present context, they should be extended to inhomogeneous chemical compositions. For instance, MacDonald \& Vennes (1991) found that the diffusive equilibrium might still be reached above the convectively mixed region.

\section{SPECTROSCOPIC DATA FROM THE SDSS AND OTHER SOURCES}

Our survey is based on the analysis of optical spectra, drawn primarily from the SDSS database, with the aim of uncovering a significant sample of chemically stratified white dwarfs. From our theoretical calculations described in the previous section, we expect these stars to have different spectral types, depending on their effective temperature and the total amount of hydrogen present in their envelope. We are, thus, searching for hybrid spectra simultaneously showing traces of hydrogen and helium. We describe below the results of our search, including additional spectra that were included for the purpose of our model atmosphere analysis.

In the original spectral classification proposed by Sion et al. (1983), the first uppercase letter after the D (for degenerate) indicates the primary spectral type, based on the dominant absorption features (DA, DB, DC, DO, DZ, and DQ; see Table 2 of Sion et al.), whereas the second uppercase letter denotes weaker or secondary spectroscopic features (e.g., a DB showing calcium features is a DBZ). In their proposed classification, DA white dwarfs show only hydrogen lines,

\footnotetext{
3 We loosely define a significant convection zone as a region where the convective flux carries a fraction larger than $10^{-7}$ of the total stellar flux, over at least three depth points, and in a region where the abundance gradient varies by more than $5 \%$.
}

and DA stars with traces of neutral or ionized helium would be called DAB and DAO stars, respectively. An exception to this rule is for DO stars with strong He II lines, for which the presence of additional $\mathrm{He} \mathrm{I}$ or $\mathrm{H}$ lines does not change the DO spectral type. Given our findings described below, we feel it would be more appropriate to refine this spectral classification and allow all uppercase letters to be present in decreasing order of line strengths. Hence, a DOB star would have a spectrum dominated by He II lines with weaker He I lines (see also Hügelmeyer et al. 2006 for a similar use), whereas a DAOB star would have a spectrum dominated by hydrogen lines, with weaker He II lines and even weaker He I lines, etc. We adopt this scheme here. Note that, because of the degeneracy of He II lines with the hydrogen Balmer lines, we refrain from classifying an object as a DOBA star. Indeed, it is difficult to distinguish a DOBA white dwarf from an ordinary DOB star, without a full model atmosphere analysis.

\subsection{Improved Spectrum for PG 1305-017}

As discussed in the Introduction, PG 1305-017 was the only DAO white dwarf-or a DAOB star, according to our refined spectral classification-analyzed by Bergeron et al. (1994) that could be interpreted as a chemically stratified atmosphere. Their spectrum was obtained in March 1992 at the Kitt Peak National Observatory $2.1 \mathrm{~m}$ telescope, using the Gold Spectrograph CCD Camera equipped with a FORD 3K chip and a $4 . .5$ slit, along with the 600 lines $\mathrm{mm}^{-1}$ grating blazed at $\lambda 4400$ in first order, providing a spectral coverage of $\lambda \lambda 3650-5140$ at an intermediate resolution of $\sim 8 \AA$ FWHM, and an achieved signal-to-noise ratio of $\mathrm{S} / \mathrm{N} \sim 80$. The quality of that spectrum was sufficient at the time to assess the stratified nature of PG 1305-017, but because this was the only stratified white dwarf known prior to our survey, we decided to obtain a much higher-quality spectrum in order to confirm its nature beyond any reasonable doubt with our improved theoretical framework. We have, thus, secured a new spectrum using the Steward Observatory $2.3 \mathrm{~m}$ telescope at Kitt Peak, equipped with the $\mathrm{B} \& \mathrm{C}$ spectrograph and a 400 lines $\mathrm{mm}^{-1}$ grating blazed at $\lambda 4889$, with a 2 ". 5 slit, providing a spectral coverage of $\lambda \lambda 3600-6900$ at an intermediate resolution of $\sim 7 \AA$ FWHM. The combined spectrum is the result of 27 exposures of 1400-1450 s each, taken during multiple nights between 2014 April and June, resulting in a total integration time of over $10.5 \mathrm{hr}$, and $\mathrm{S} / \mathrm{N} \sim 325$ over the same range as the earlier spectrum. This much-improved spectrum of PG $1305-017$ is displayed in Figure 7, along with the older observation of Bergeron et al. (1994). We chose this particular flux scale to emphasize how shallow all the absorption features are (i.e., less than $\sim 10 \%$ ).

\subsection{Spectra from the SDSS}

Most of the spectra in our sample come from the SDSS spectroscopic database, taken with the Apache Point Observatory $2.5 \mathrm{~m}$ telescope in New Mexico. These provide a spectral coverage of $3800-9200 \AA$ at an intermediate resolution of 2-3 $\AA$ FWHM. Because of the fixed exposure time of each plate, the $\mathrm{S} / \mathrm{N}$ of these spectra depends directly on the magnitude of the star, and thus varies considerably from object to object, roughly from $\mathrm{S} / \mathrm{N} \sim 10$ to as high as $\sim 100$, in some rare instances. We searched the SDSS catalogs of spectroscopically identified white dwarfs from the Data Release 7 


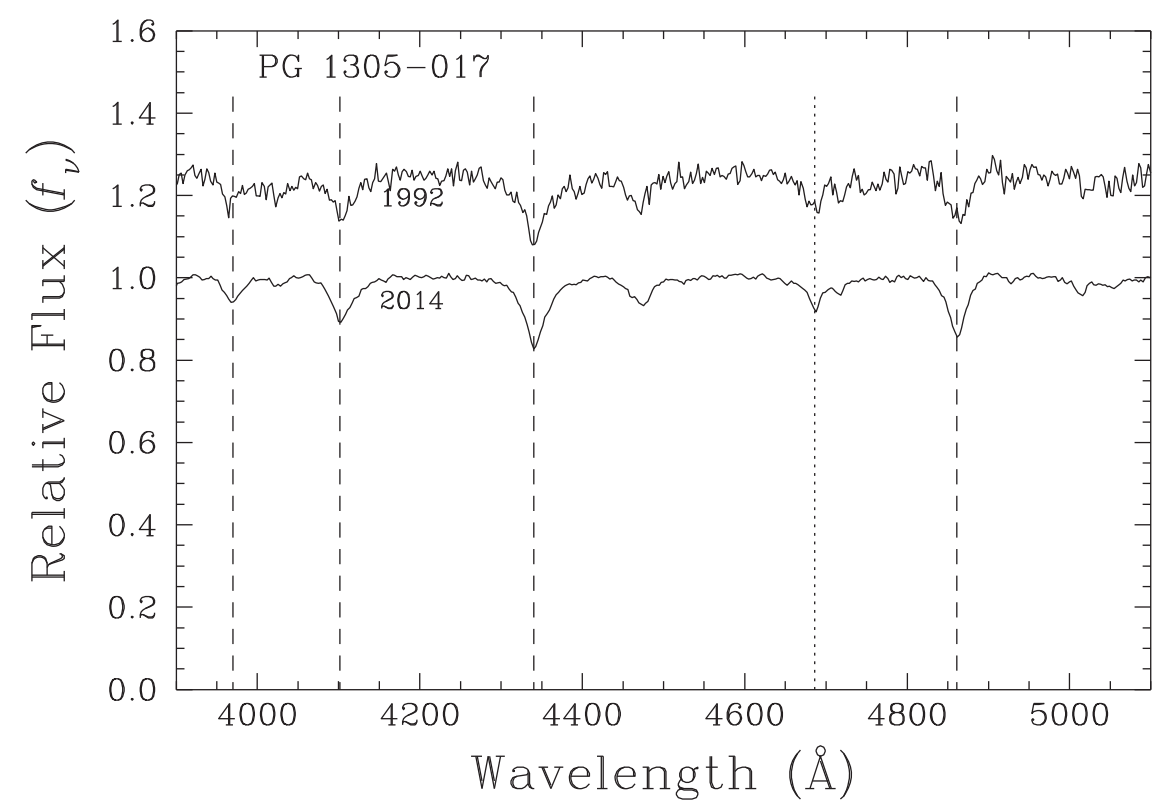

Figure 7. Optical spectra for PG 1305-017 obtained by Bergeron et al. (1994, top) and by us (bottom); both spectra are normalized to a continuum set to unity and offset vertically by 0.25 for clarity. Here and in the following figures, we show by dashed lines the location of the hydrogen Balmer line series, and by a dotted line the location of the He II $\lambda 4686$ absorption feature.

(DR7; Kleinman et al. 2013), DR10 (Kepler et al. 2015), and DR12 (Kepler et al. 2016), for a combined sample of $~ 38,000$ white dwarf spectra.

We restricted our search of hybrid white dwarfs to SDSS spectra with $S / N>20$. All spectra were first normalized to a continuum set to unity, and then, by fitting Gaussian profiles, we searched for spectra with $\mathrm{H} \beta, \mathrm{H} \gamma$, and He II $\lambda 4686$ deeper than $\sim 0.03$ for all of these absorption features. This simple procedure resulted in a fairly large sample of $\sim 1000$ objects with more or less hybrid spectra. We then inspected each spectrum visually, and removed from that sample all DA, DB, and DO stars. It is important to note that, at this point, the sample is not complete and there are some subjective biases involved. We also found duplicate spectra for the same star and only kept the most recent one. This selection resulted in a reduced sample of 49 hybrid spectra, including $22 \mathrm{DAO}, 3$ DAOB, 2 DOAB, 12 DOB, 6 DBO stars, and also 2 DAB and 2 DBA stars that were kept in our sample because a quick spectroscopic fit indicated that they were fairly hot ( $\left.T_{\text {eff }} \gtrsim 30,000 \mathrm{~K}\right)$. Traces of He II indicate $T_{\text {eff }} \gtrsim 40,000 \mathrm{~K}$, whereas the simultaneous presence of He I lines suggests that the effective temperature is not high enough to completely ionize helium, which occurs around $60,000 \mathrm{~K}$. Thus, the majority of our sample lies in the vicinity of the blue edge of the DB gap at $T_{\text {eff }} \sim 45,000 \mathrm{~K}$.

We show in Figures 8 and 9 the hybrid spectra we identified in the SDSS. These spectra illustrate the large diversity of features - in particular, the relative strength of hydrogen and helium lines that could only be uncovered in a large spectroscopic survey such as the SDSS. A large fraction of our sample is dominated by DAO stars, probably all of which have chemically homogeneous compositions according to the analyses of Bergeron et al. (1994) and Gianninas et al. (2010).

\subsection{Follow-up Spectroscopy with Gemini Observatory}

Three objects in the SDSS sample-J0033+1422 (WD 0031 +141), J0342-0722 (WD 0340-075), and J2351+0108 (PB
5559) - were identified as being potentially chemically stratified in our preliminary spectroscopic analysis. In order to improve the quality of the spectrum for these three candidates, we obtained four hours of observation on the Gemini-South Observatory $8 \mathrm{~m}$ telescope in Chile. The Hamamatsu CCD and the B600 G5323 600 lines $\mathrm{mm}^{-1}$ grating were used with a 1!!0 slit, providing a spectral coverage from 3800 to $6600 \AA$, with a spectral resolution of $5 \AA$ FWHM. Six exposures of 600 s per star were taken at a central wavelength of 520, 523, and $526 \mathrm{~nm}$, to account for the gaps in the detector and remove the cosmic rays. The resulting $\mathrm{S} / \mathrm{N}$ is $\sim 100$. Unfortunately, a problem with the \#5 amplifier of the CCD, as identified by the Gemini staff, occurred during the observation of two of our targets, which led to a pixel saturation between columns 1000 and 1300 , corresponding to $\lambda \sim 4950-5350 \AA$ on the reduced spectra. The CCD was eventually fixed for the observation of our third target (WD 0031+141).

The requested Gemini standard calibration contained only one flux standard object, which proved to be insufficient for an adequate flux calibration. Instead, we relied on the corresponding SDSS spectrum of each object, properly flux calibrated, to calibrate our Gemini spectroscopic observations. We obtained a flux calibration function by simply dividing the SDSS spectrum by the Gemini spectrum, and then smoothed the result using a Savitzky-Golay filter (Savitzky \& Golay 1964), which simply fits successive subsets of adjacent data points with a low-degree polynomial by a linear least squares method. The reduced Gemini spectra are displayed in Figure 10.

\subsection{SDSS J074538.17+312205.3}

Eisenstein et al. (2006b) presented a detailed model atmosphere analysis of $28 \mathrm{DO}$ and DB stars identified in the SDSS. One of their targets, SDSS J074538.17+312205.3, is of particular interest here, because its spectrum shows traces of both hydrogen and helium - the only DBAO in our sample analyzed below. The spectrum for this object, obtained with the 


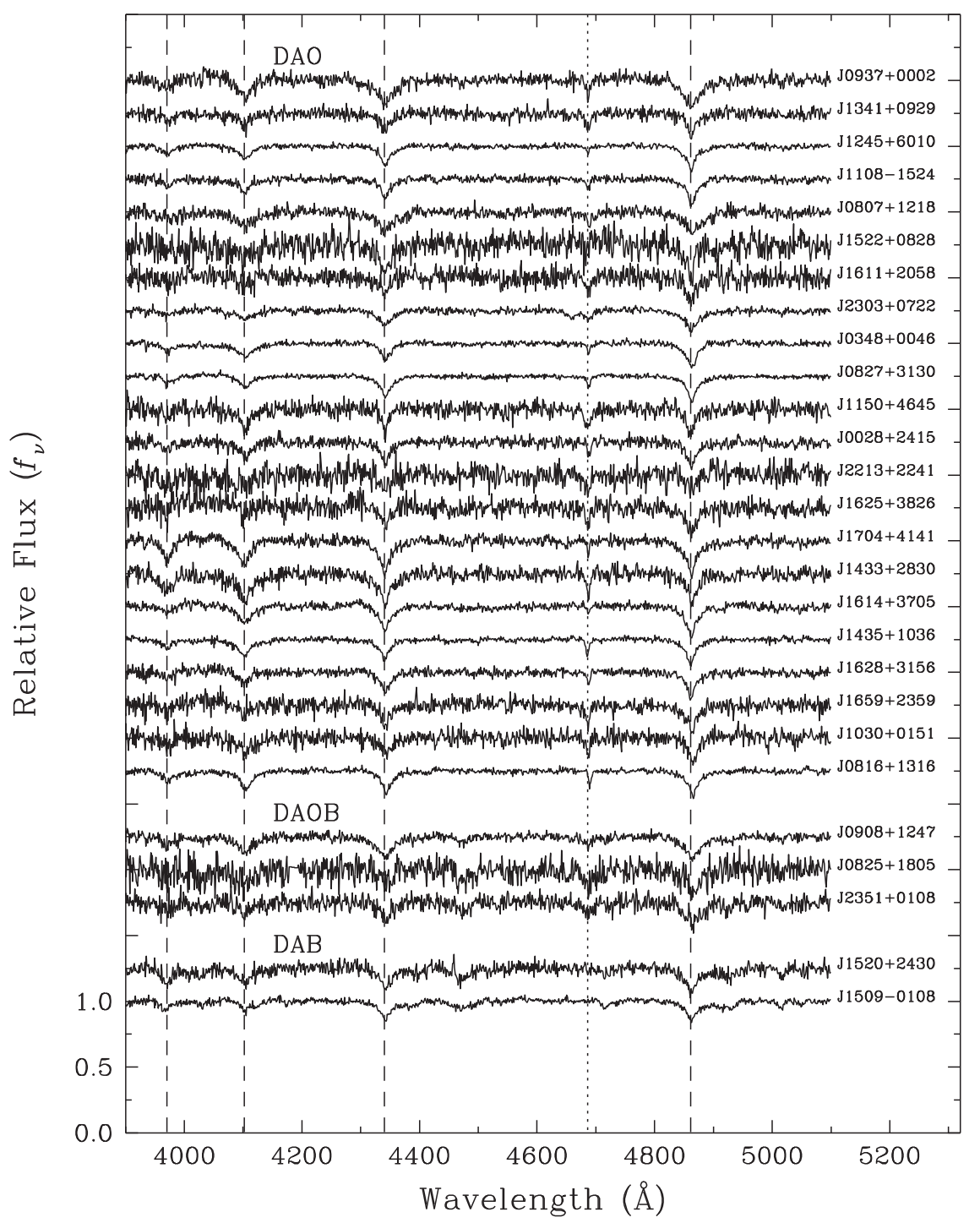

Figure 8. Optical spectra of hybrid white dwarfs in the SDSS with spectra dominated by hydrogen lines that also show traces of He II and/or He I lines (i.e., DAO, DAOB, and DAB stars).

MMT $6.5 \mathrm{~m}$ telescope on Mt. Hopkins-kindly provided to us by D. Eisenstein-is displayed in Figure 11. More interestingly, Eisenstein et al. failed to fit this star with homogeneous models in a satisfactory manner (see their Figure 1), and they even proposed that this white dwarf might have a chemically stratified atmosphere.

\section{MODEL ATMOSPHERE ANALYSIS}

In this section, we present the results of our spectroscopic analysis, using both our chemically homogeneous and stratified model atmosphere grids. In all cases, both the observed and model spectra (convolved with the appropriate Gaussian instrumental profile) are normalized to a continuum set to unity, and the atmospheric parameters $-T_{\text {eff }}, \log g$, and either $N(\mathrm{He}) / N(\mathrm{H})$ for the homogeneous solution or $\log q_{\mathrm{H}}$ for the stratified solution ${ }^{4}$ - are determined using the nonlinear leastsquares minimization method of Levenberg-Marquardt (Press

\footnotetext{
4 As discussed in Section 2.1, because the value of $\log g$ significantly affects the relative strength of hydrogen and helium lines for a given value of $q_{\mathrm{H}}$, it is actually the parameter $Q \equiv 3 \log g+2 \log q_{\mathrm{H}}$ that is being fitted rather than $\log q_{\mathrm{H}}$ directly.
}

et al. 1986), whereas the uncertainties of each atmospheric parameter are obtained directly from the covariance matrix (see Bergeron et al. 1992 for details).

\section{1. $P G 1305-017$}

The best fit to our improved spectrum of PG 1305-017 is displayed in Figure 12. The atmospheric parameters obtained here for both the homogeneous and stratified models are entirely consistent with those reported in Table 3 of Bergeron et al. (1994). Hence, despite the various improvements included in our current model grid, the spectroscopic solutions have not changed significantly, and the results and conclusions reached in the analysis of Bergeron et al. remain valid. A most significant improvement over this earlier analysis, however, is the spectacular fit achieved here with the stratified models compared to the homogeneous solution. In contrast with the results displayed in Figure 3 of Bergeron et al., there is now absolutely no doubt that PG $1305-017$ possesses a chemically stratified atmosphere. This clearly demonstrates that the floatup model, where a thin hydrogen layer floats in diffusive equilibrium on top of the helium envelope, does occur in 


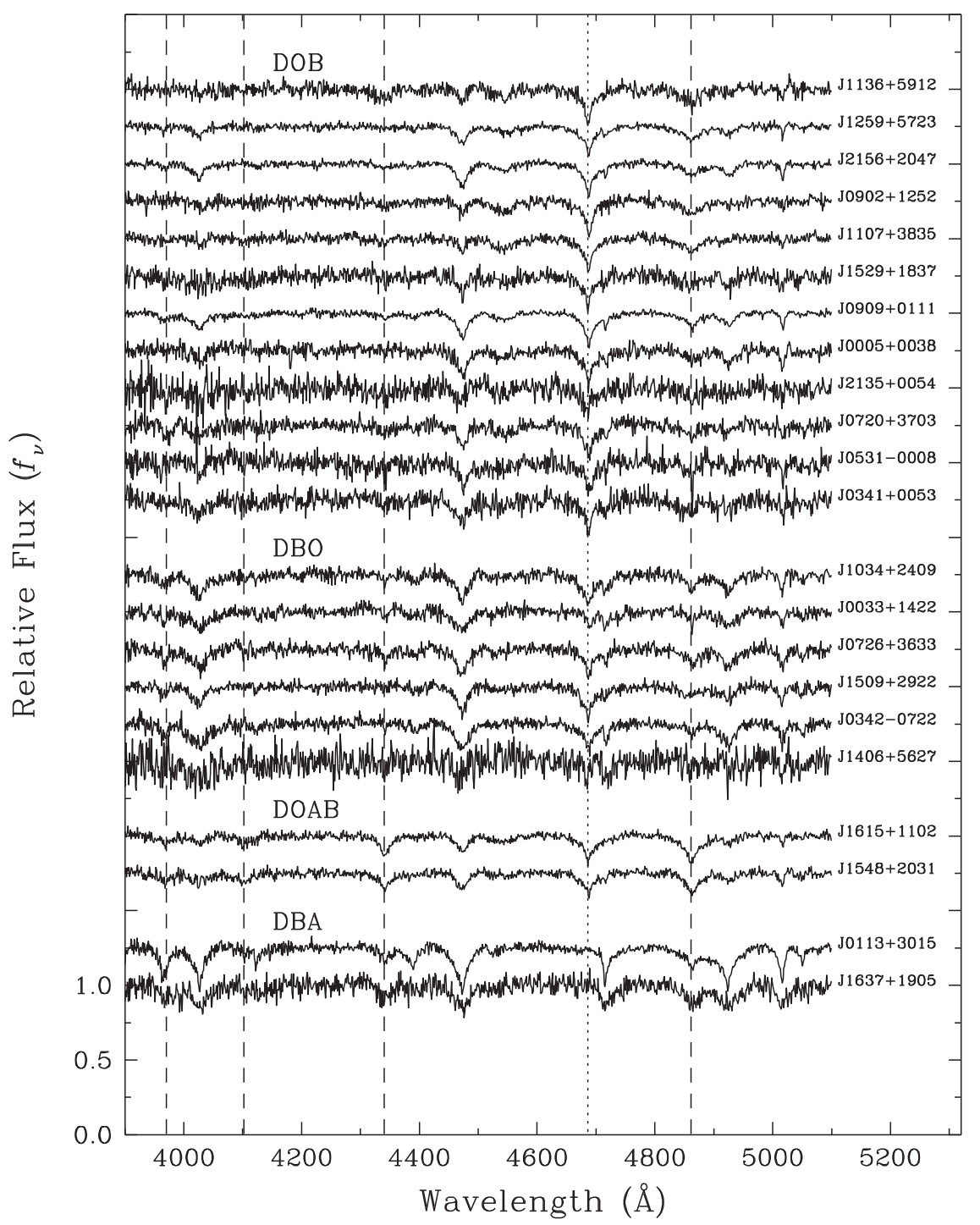

Figure 9. Optical spectra of hybrid white dwarfs in the SDSS with spectra dominated by He II lines (DO) or He I lines (DB) that also show hydrogen lines, and sometimes two simultaneous ionization stages of helium with different relative strength.

nature. The atmospheric parameters for PG 1305-017 $-T_{\text {eff }}=44,890 \mathrm{~K}, \quad \log g=7.89, \quad$ and $\log q_{\mathrm{H}} \sim-16$-are almost identical to one of the abundance profiles displayed in Figure 1, which reveals that hydrogen and helium are present in about equal amounts at the photosphere. Furthermore, according to the results shown in Figure 6, PG 1305-017 is located slightly hotter than the region where convection may play a role in homogenizing the chemical stratification, according to our own criteria. Because PG 1305-017 is clearly stratified, we are confident that our criteria used to establish the importance of convective mixing are physically sound.

\subsection{Discriminating Homogeneous and Stratified Atmospheres}

Before proceeding with the spectroscopic analysis of our sample, we must first investigate whether the homogeneous and stratified solutions can be easily distinguished throughout the entire parameter space we are exploring in our investigation. Indeed, despite the fact that the stratified solution provides a much better fit to the spectrum of PG 1305-017 than the homogeneous solution (see Figure 12), the differences remain somewhat subtle-restricted to the He I $\lambda 4471$ and He II $\lambda 4686$ absorption features in the case of PG 1305-017-and the very high $\mathrm{S} / \mathrm{N}$ of about 325 that we achieved for this object is a luxury we cannot afford for most white dwarfs in our sample.

We show, in Figure 13, the results of an experiment where we fitted our grid of homogeneous model spectra at $\log g=8$ using our stratified models. The regions where the standard deviations are the largest represent the locations in temperature and heliumto-hydrogen abundance ratio where the homogeneous and stratified solutions can be easily discriminated. For instance, white dwarfs with homogeneous solutions at $\log N(\mathrm{He}) /$ $N(\mathrm{H})<-2$ at all temperatures also have stratified solutions that are practically indistinguishable. A similar conclusion is reached for white dwarfs with $\log N(\mathrm{He}) / N(\mathrm{H})>1$ but for $T_{\text {eff }} \gtrsim 45,000 \mathrm{~K}$. However, objects with comparable amounts of hydrogen and helium, $\log N(\mathrm{He}) / N(\mathrm{H}) \sim 0 \pm 1$, can be easily distinguished-in particular, at low temperatures $\left(T_{\text {eff }} \lesssim\right.$ $45,000 \mathrm{~K}$ ), which is exactly the case for PG 1305-017. One of the best examples in our sample is for J1509-0108, displayed in Figure 14, where the stratified solution not only provides a better fit than the homogeneous solution, but the atmospheric parameters are significantly different as well. In this particular white dwarf, the hydrogen lines are so weak that the only way for the 


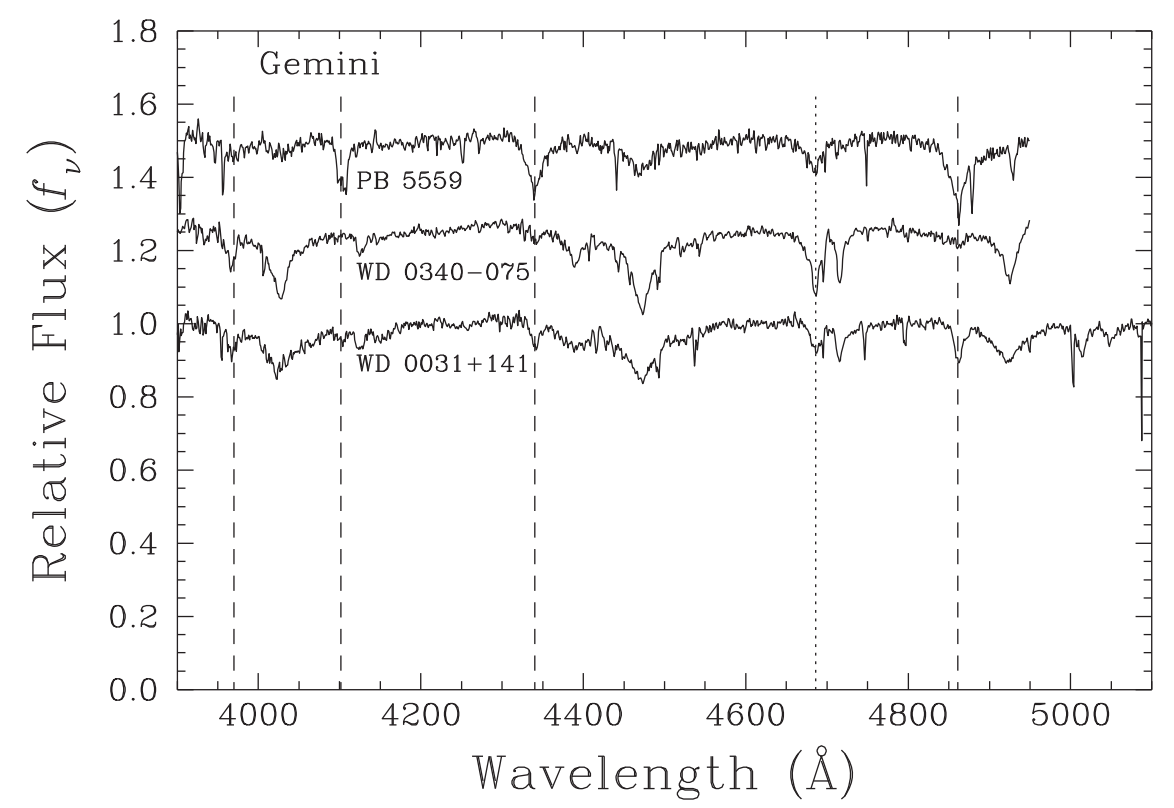

Figure 10. Optical spectra of three stratified white dwarf candidates obtained with the Gemini-South Observatory $8 \mathrm{~m}$ telescope in Chile, which had been identified in our preliminary model atmosphere analysis of the corresponding SDSS spectra.

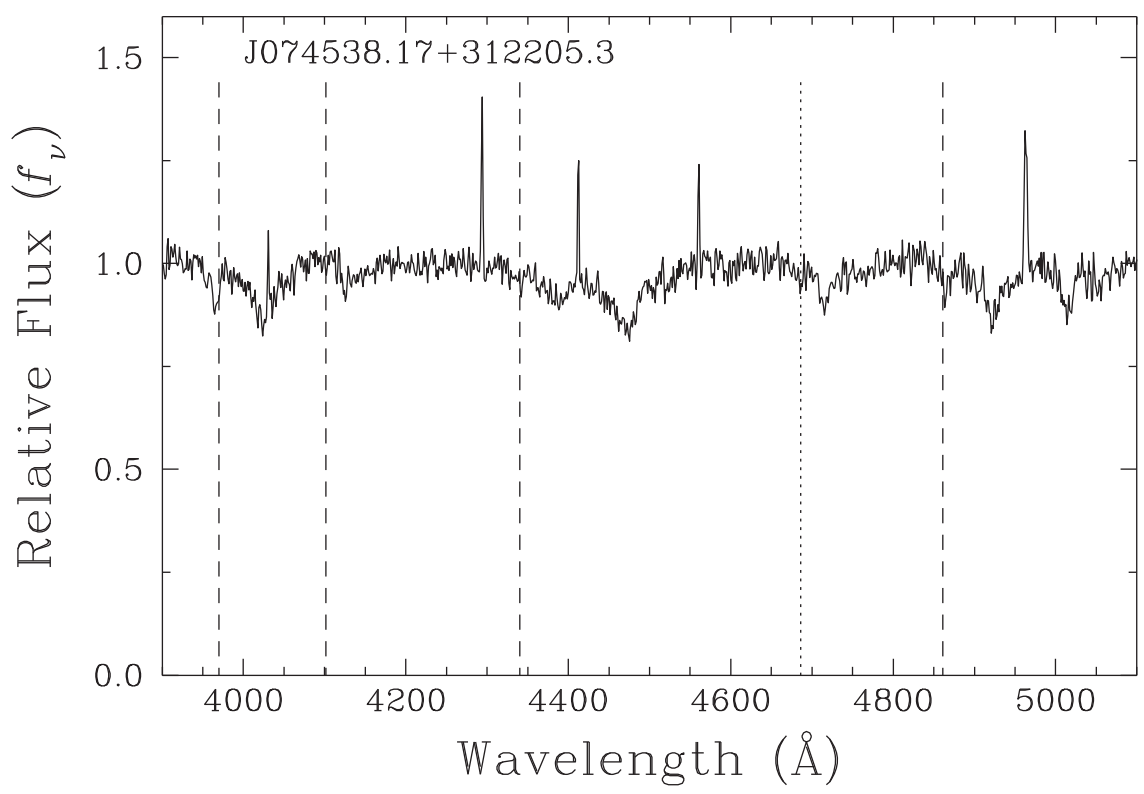

Figure 11. Optical spectrum of SDSS J074538.17+312205.3 obtained with the Blue Channel spectrograph mounted on the MMT $6.5 \mathrm{~m}$ telescope, kindly provided to us by D. Eisenstein. The 500 lines $\mathrm{mm}^{-1}$ grating was used with a $1^{\prime \prime} 5$ slit, providing a spectral coverage from 3750 to $6850 \AA$ at a resolution of $6 \AA$ FWHM.

homogeneous models to reproduce the observed Balmer lines is to push the effective temperature to $\sim 47,000 \mathrm{~K}$, predicting a strong He II $\lambda 4686$ absorption feature, which is simply not detected. In contrast, the stratified solution for J1509-0108 is found at a much lower temperature $\left(T_{\text {eff }} \sim 30,000 \mathrm{~K}\right)$ where helium is neutral; there simply is no homogeneous solution near that temperature. We also note that our stratified solution is in excellent agreement with the photometric temperature we estimated at $T_{\text {eff }} \sim 30,000 \mathrm{~K}$ based on the available ugriz photometry. Hence, stratified white dwarfs may exist at very low effective temperatures, and their hybrid spectra may not necessarily show He II $\lambda 4686$ (as in DAO or DOA stars).

\subsection{Evidence for Chemical Stratification in White Dwarfs}

In addition to PG 1305-017 and J1509-0108 discussed above, we identified several other white dwarfs in our sample that show evidence for chemical stratification. In Figure 15, we revisit the spectrum of SDSS J074538.17+312205.3 (J0745 +3122 ) analyzed by Eisenstein et al. (2006b, see their Figure 1), who suggested that the poor fit to their optical MMT spectrum could be the result of chemical stratification. Our fit to the same spectrum with the homogeneous models displayed here shows the same problem-in particular, for the He I $\lambda 4471$ line, but also for the overall spectrum. Our fit with stratified models, however, is in much better agreement-with 


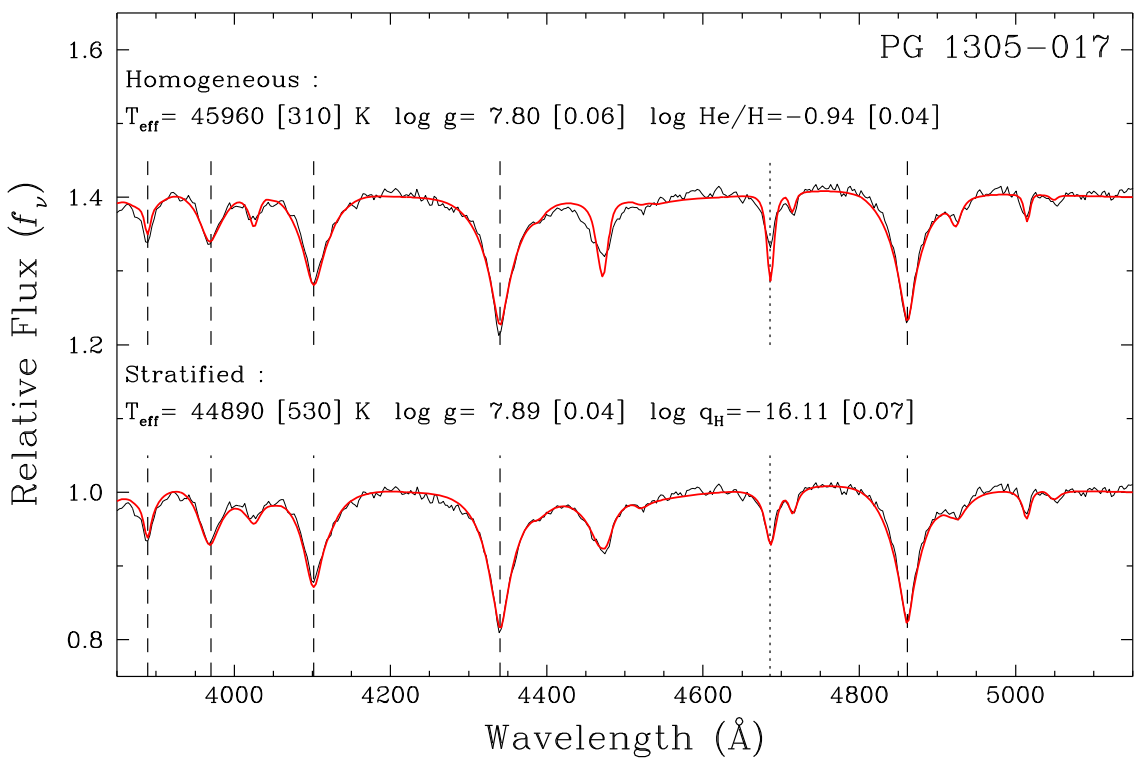

Figure 12. Our best fits (red) to the optical spectrum (black) of PG 1305-017, using homogeneous (top) and stratified (bottom) composition models; the atmospheric parameters of each solution are given in the figure. Both the observed and theoretical spectra are normalized to a continuum set to unity; the top spectrum is shifted by a factor of 0.4 for clarity. Here, a best fit is clearly achieved with stratified models.

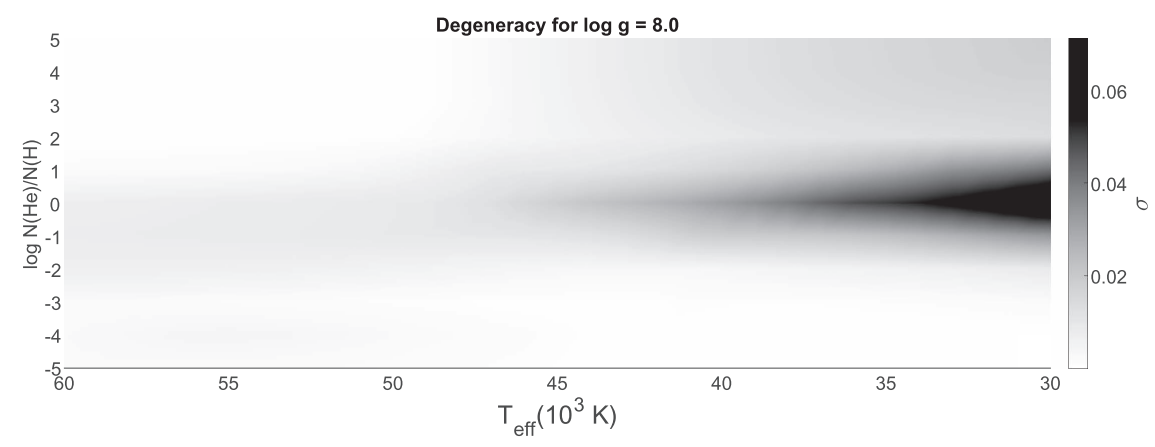

Figure 13. Standard deviations $\sigma$ obtained from the best spectroscopic fits of our homogeneous model grid at $\log g=8$, using our stratified model grid, as a function of effective temperature and helium-to-hydrogen abundance ratio. The darkest areas in this figure indicate regions in $T_{\text {eff }}$ and $N(\mathrm{He}) / N(\mathrm{H})$ where the homogeneous and stratified solutions can be easily distinguished, whereas white areas correspond to regions where both solutions are practically indistinguishable.

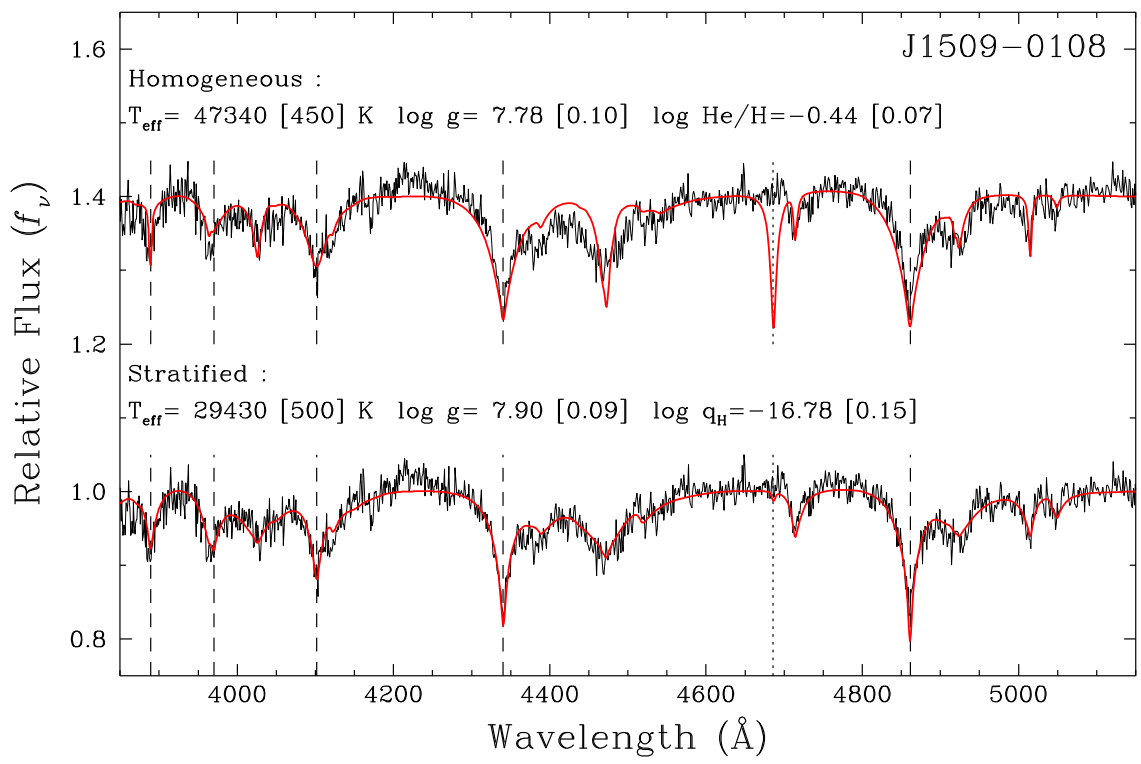

Figure 14. Same as Figure 12 but for J1509-0108. 


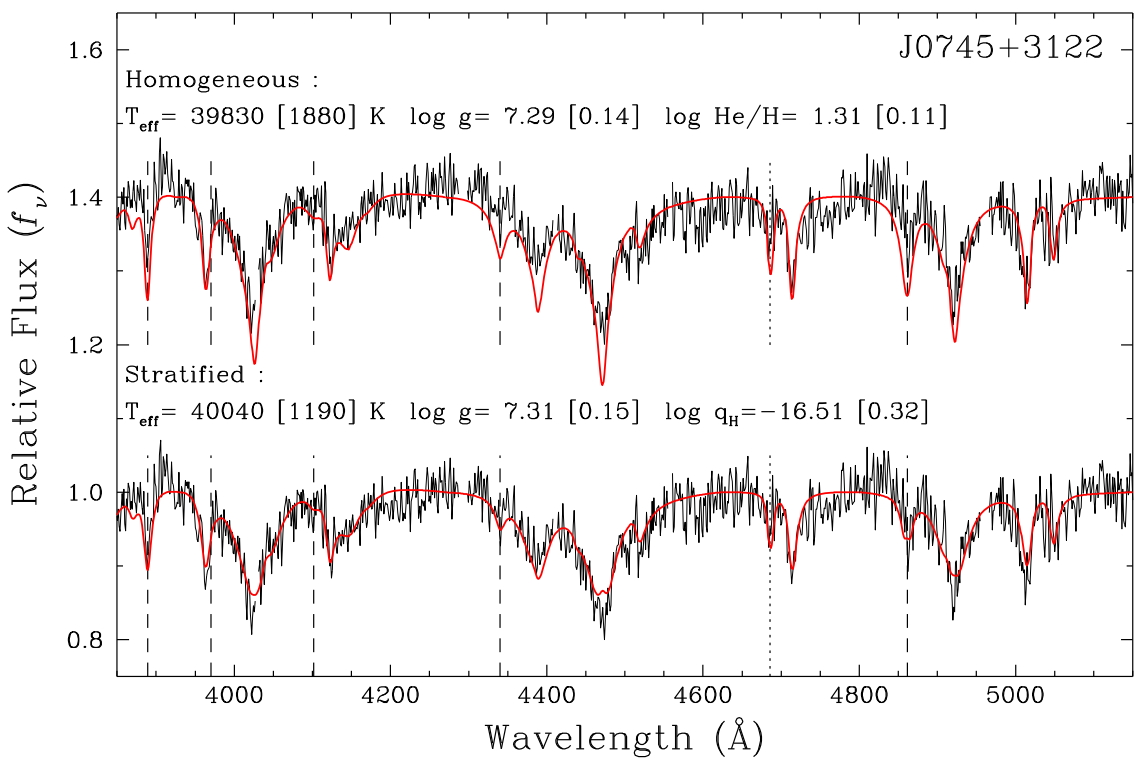

Figure 15. Same as Figure 12 but for J0745+3122. The spectrum is from Eisenstein et al. (2006b).

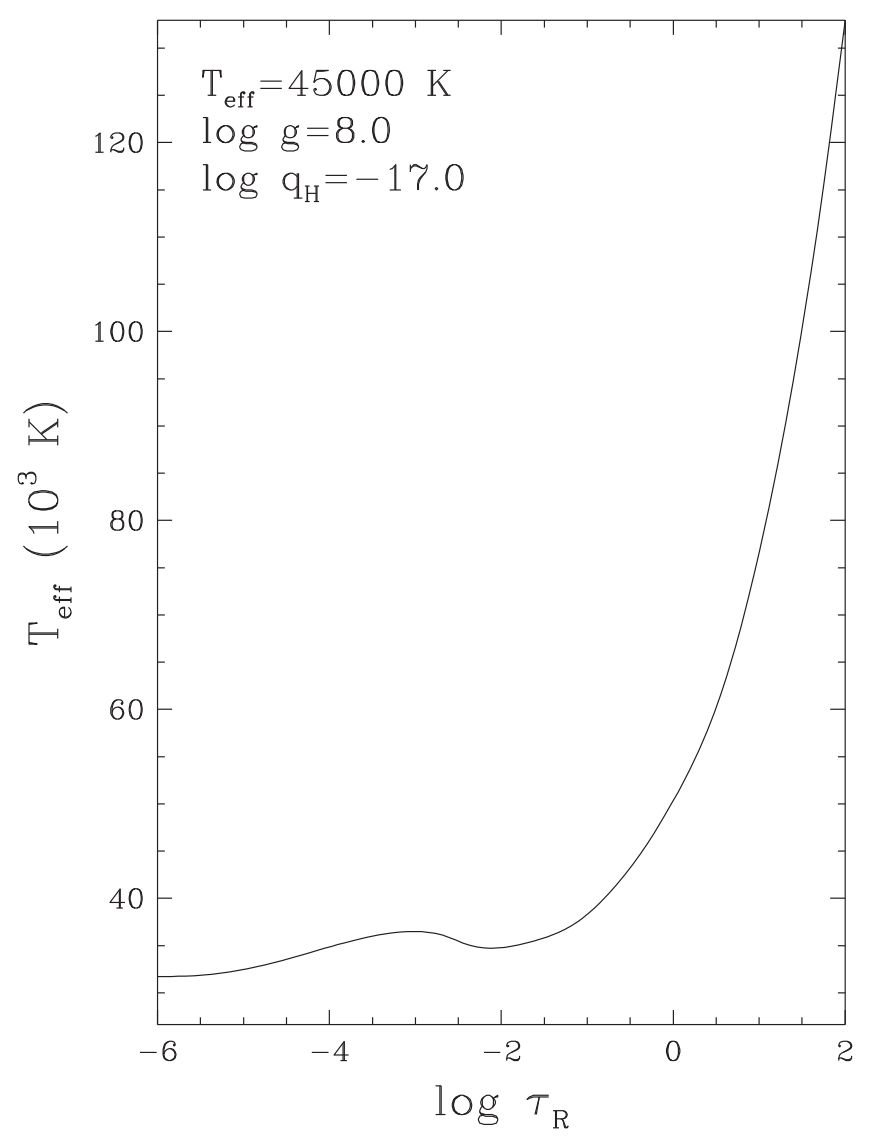

Figure 16. Temperature structure as a function of Rosseland optical depth in a stratified model from our LTE grid that shows a temperature inversion near the surface.

the notable exception of the line core of He I $\lambda 4471$, for which the model appears almost in emission (other cases can be found below). We traced back this particular feature to a small temperature inversion that occurs in some of our models (see Figure 16 for one example). Such temperature inversions may occur even in LTE models (see, e.g., Dumont \& Heidmann 1973) for conditions related to a sudden change of opacity in the upper atmosphere, as is the case here for stratified models. However, at the small optical depth where this temperature inversion occurs, $\tau_{R} \sim 10^{-3}$ according to Figure 16, the line cores formed there should probably be treated in NLTE. Despite these small discrepancies, the stratified solution for $\mathrm{J} 0745+3122$ offers a much better fit to the overall spectrum, particularly to the regions near the hydrogen lines $\mathrm{H} \beta$ and $\mathrm{H} \gamma$.

In Figure 17, we show additional convincing cases of chemically stratified white dwarfs, all of which fall in the $40,000-50,000 \mathrm{~K}$ temperature range where the majority of nonDA stars are expected to turn into DA stars. For both J0033 +1422 (WD 0031+141) and J2351+0108 (PB 5559), we show our fits to the SDSS spectra (Figures 17(c) and (f), respectively) as well as the fits to our Gemini spectra (Figures 17(d) and (g), respectively). The case of $\mathrm{J} 0033+1422$ is worth discussing. Here, our fits to the SDSS and Gemini spectra using homogeneous models yield a poor fit to the He II $\lambda 4686$ line, compared to the fits using stratified models. One can also notice that the helium-to-hydrogen abundance ratio varies by more than 3 dex between the two homogeneous solutions (the value of $\log \mathrm{He} / \mathrm{H}=5$ is actually the upper limit of our grid). At the temperature inferred here with homogeneous models $\left(T_{\text {eff }} \sim\right.$ $50,000 \mathrm{~K}$ ), both solutions are consistent with pure helium models, and the absorption feature predicted near $\mathrm{H} \beta$ is in fact a He II line. In contrast, the stratified solutions yield a much lower temperature near $43,000 \mathrm{~K}$, and hence, a much weaker He II $\lambda 4686$ line. In this case, the absorption feature near $\mathrm{H} \beta$ is due to hydrogen; an absorption feature from $\mathrm{H} \gamma$ is also predicted-and observed-as opposed to the homogeneous models.

Additional fits to stratified white dwarf candidates, which are not as convincing as those shown previously, are displayed in Figure 18, again using SDSS and Gemini spectra. One could argue that, for these stars, both the homogeneous and stratified solutions are equally acceptable. However, all of those stars are hot-although not hot enough to sustain a stellar wind-and their atmospheres are completely radiative. Thus, the only viable solution from an astrophysical point of view is that their atmospheres are chemically stratified. There exists no other 


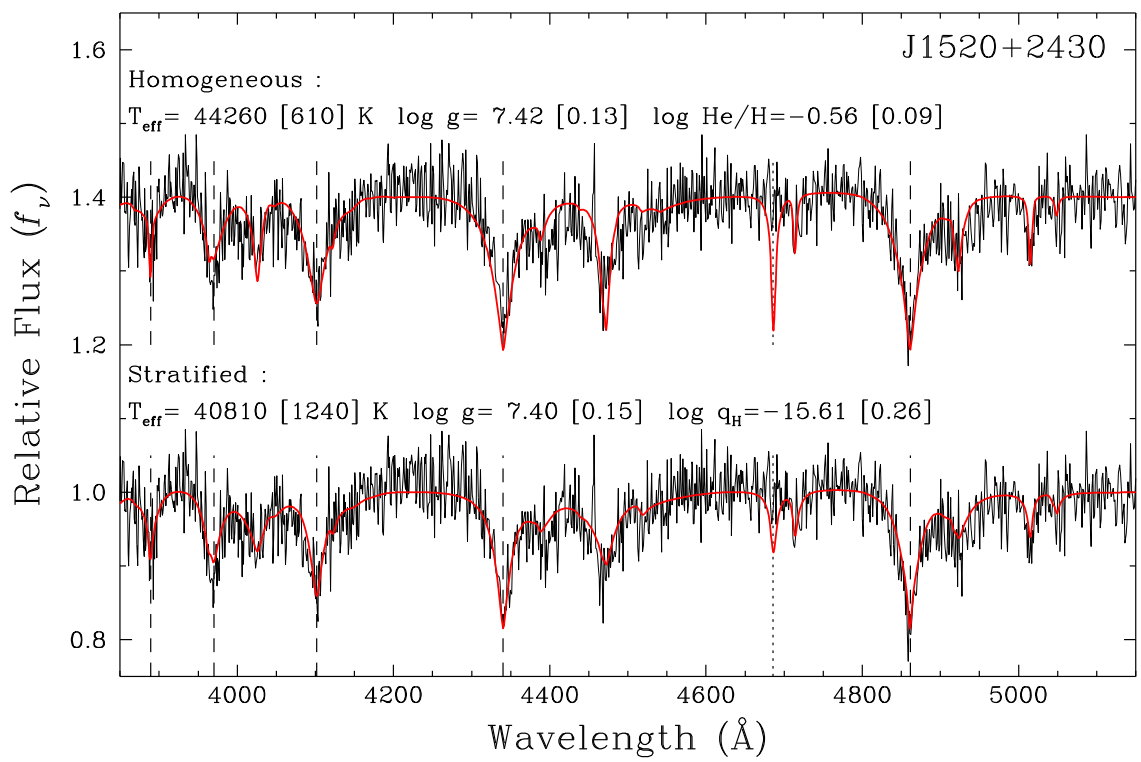

Figure 17. Spectroscopic fits for white dwarfs from the SDSS and Gemini (WD 0031+141, PB 5559) that bear the signature of a stratified atmosphere. The spectrum of $\mathrm{J} 1406+5627$ has been filtered to reduce the noise level. (An extended version of this figure is available.)

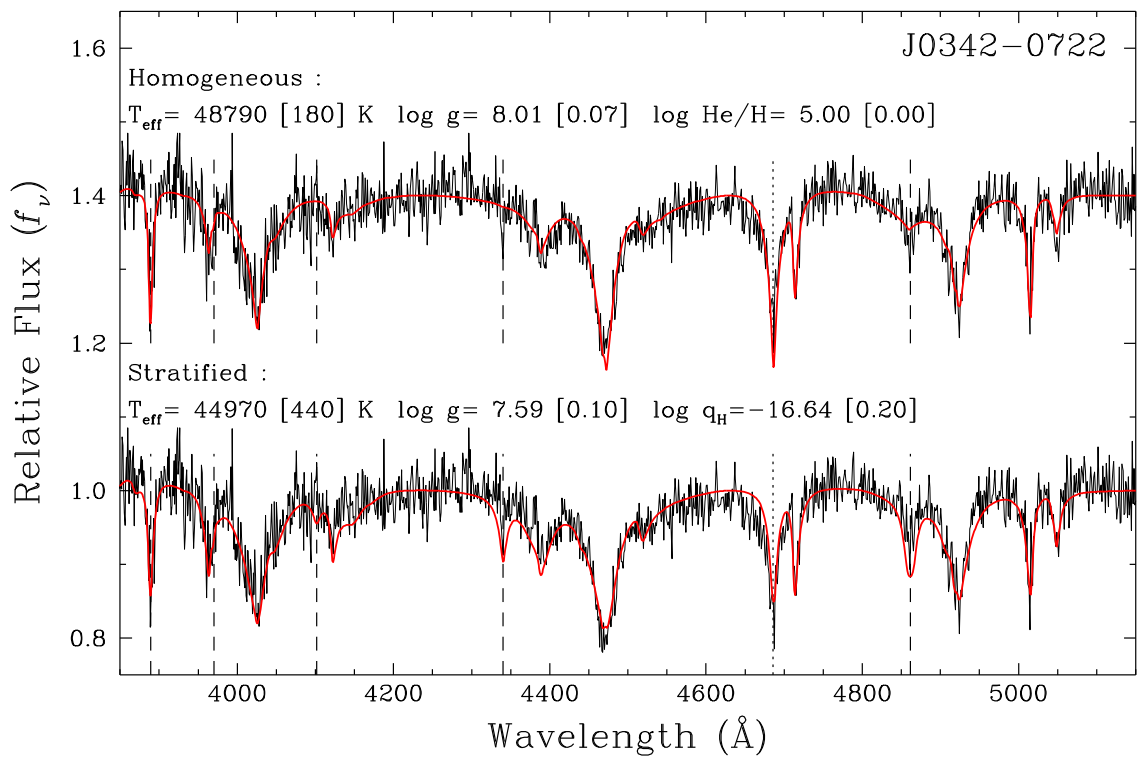

Figure 18. Spectroscopic fits for white dwarfs from the SDSS and Gemini (WD 0340-075) whose solutions cannot be distinguished. The spectrum of J0825+1805 has been filtered to reduce the noise level. (An extended version of this figure is available.)

physical mechanism that could account for the simultaneous presence of hydrogen and helium in their photosphere. Moreover, we have explicitly verified in all cases that for both the homogeneous and stratified solutions, the spectrum does show hydrogen lines, as opposed to other cases discussed above.

\subsection{Cool DOB and DBO White Dwarfs}

For hot, helium-dominated atmospheres with either extremely low hydrogen abundances or very thin hydrogen layers, the only way to infer the presence of hydrogen is through a detailed model atmosphere analysis, because the hydrogen lines coincide with those of He II. We show, in Figure 19, our spectroscopic fits for cool DOB and DBO white dwarfs in our sample whose homogeneous and stratified solutions cannot be distinguished. In all cases displayed here, the spectroscopic solutions are consistent with pure helium compositions. In particular, the absorption features predicted near $\mathrm{H} \beta$ and $\mathrm{H} \gamma$ are actually $\mathrm{He}$ II lines. For the stratified solutions, the hydrogen layers inferred here are so thin that the line forming region is totally embedded in the helium-dominated layer. Hence, these stars have little or no hydrogen whatsoever. They are likely to evolve as pure helium DB stars in the region of the DB deficiency, i.e., in the 30,000-45,000 K temperature range, and continue as cooler DB stars with no hydrogen features, such as those identified in the analysis of Bergeron et al. (2011).

\subsection{Chemically Homogeneous White Dwarfs}

Most of the DAO stars displayed in Figure 8 turned out to be hotter than the maximum temperature of our model grid $\left(T_{\text {eff }}=60,000 \mathrm{~K}\right)$ and were therefore discarded. These are J0028+2415, J0348+0046, J0807+1218, J0827+3130, J0902 


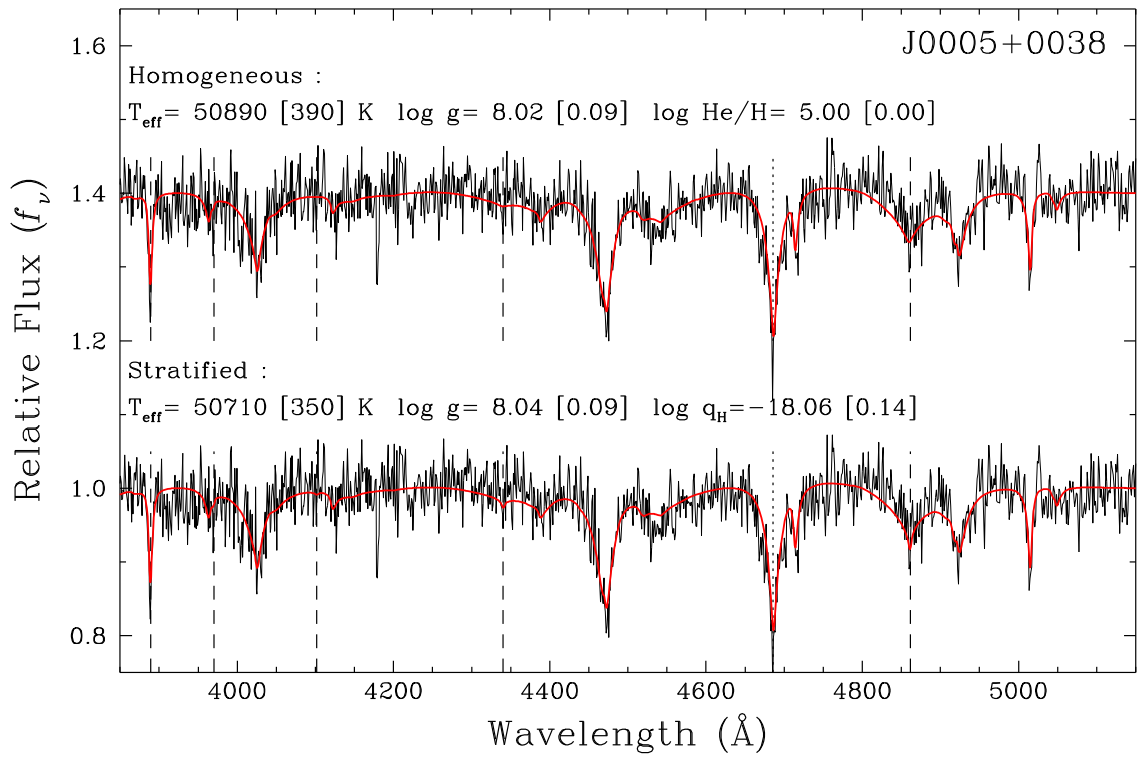

Figure 19. Spectroscopic fits for cool DOB and DBO white dwarfs from the SDSS whose homogeneous and stratified solutions cannot be distinguished. In both cases, the solutions displayed here are consistent with pure helium atmospheres, and the absorption features very near the locations of $\mathrm{H} \beta$ and $\mathrm{H} \gamma$ are $\mathrm{He}$ II lines. (An extended version of this figure is available.)

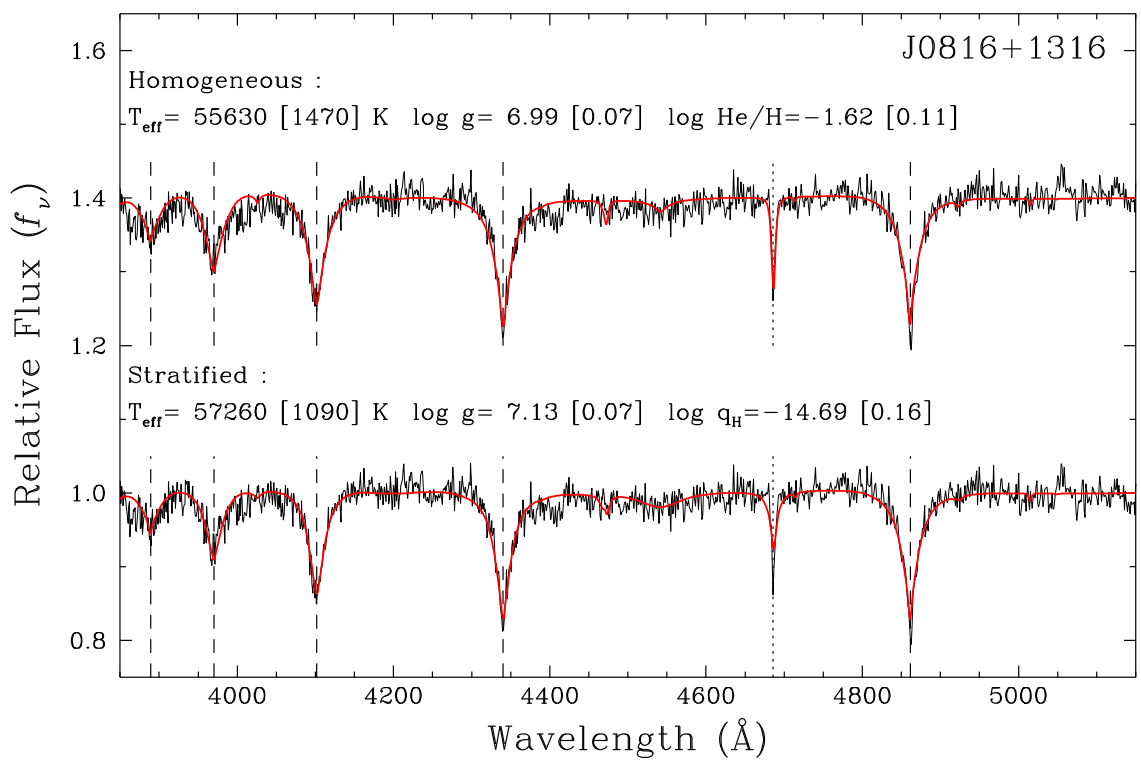

Figure 20. Spectroscopic fits for cool DAO white dwarfs from SDSS whose spectra are better fitted with homogeneous models. (An extended version of this figure is available.)

$+1252, \quad \mathrm{~J} 1030+0151, \quad \mathrm{~J} 1108-1524, \quad \mathrm{~J} 1150+4645, \quad \mathrm{~J} 1245$ $+6010, \quad \mathrm{~J} 1341+0929, \quad \mathrm{~J} 1435+1036, \quad \mathrm{~J} 1522+0828, \quad \mathrm{~J} 1611$ $+2058, \quad \mathrm{~J} 1625+3826, \quad \mathrm{~J} 1628+3156, \quad \mathrm{~J} 1659+2359, \quad \mathrm{~J} 2213$ +2241 , and $\mathrm{J} 2303+0722$. The spectroscopic fits to the five cooler DAO stars in our sample are displayed in Figure 20. In all cases, the quality of the fits rests on the He II $\lambda 4686$ line, which is better reproduced with homogeneous models, a conclusion also reached for hotter DAO stars by Bergeron et al. (1994, see their Figure 4) based on a similar analysis. With the exception of J0937+0002, all these white dwarfs have extremely low surface gravities and probably require NLTE models to achieve more accurate atmospheric parameters. Also, in most cases, the hydrogen Balmer lines, as well as the He II $\lambda 4686$ line, are much deeper than predicted. This so-called Balmer-line problem has been discussed at length in Gianninas et al. (2010) and references therein, and manifests itself as an inability to fit all the Balmer lines simultaneously with consistent atmospheric parameters. The solution to this problem, also discussed in Gianninas et al., is to include heavy elements- $\mathrm{CNO}$ in particular-in the model atmosphere calculations, whose main effect is to cool the surface temperature, leading to deeper line cores (see also Werner 1996). Note the presence of C IV $\lambda 4658$ in the spectrum of $\mathrm{J} 1704+4141$ (Figure 20(e)). It is also believed that the presence of these heavy elements is responsible for driving a weak stellar wind, which in turn could explain the presence of helium in DAO white dwarfs. This point is further discussed below. An improved modeling of the DAO stars displayed in Figure 20 is clearly outside the scope of our paper.

Finally, we show, in Figure 21, our best fits to the two DBA white dwarfs in our sample. Both objects are relatively hot, $T_{\text {eff }} \sim 30,000 \mathrm{~K}$, and their spectra are better reproduced with 

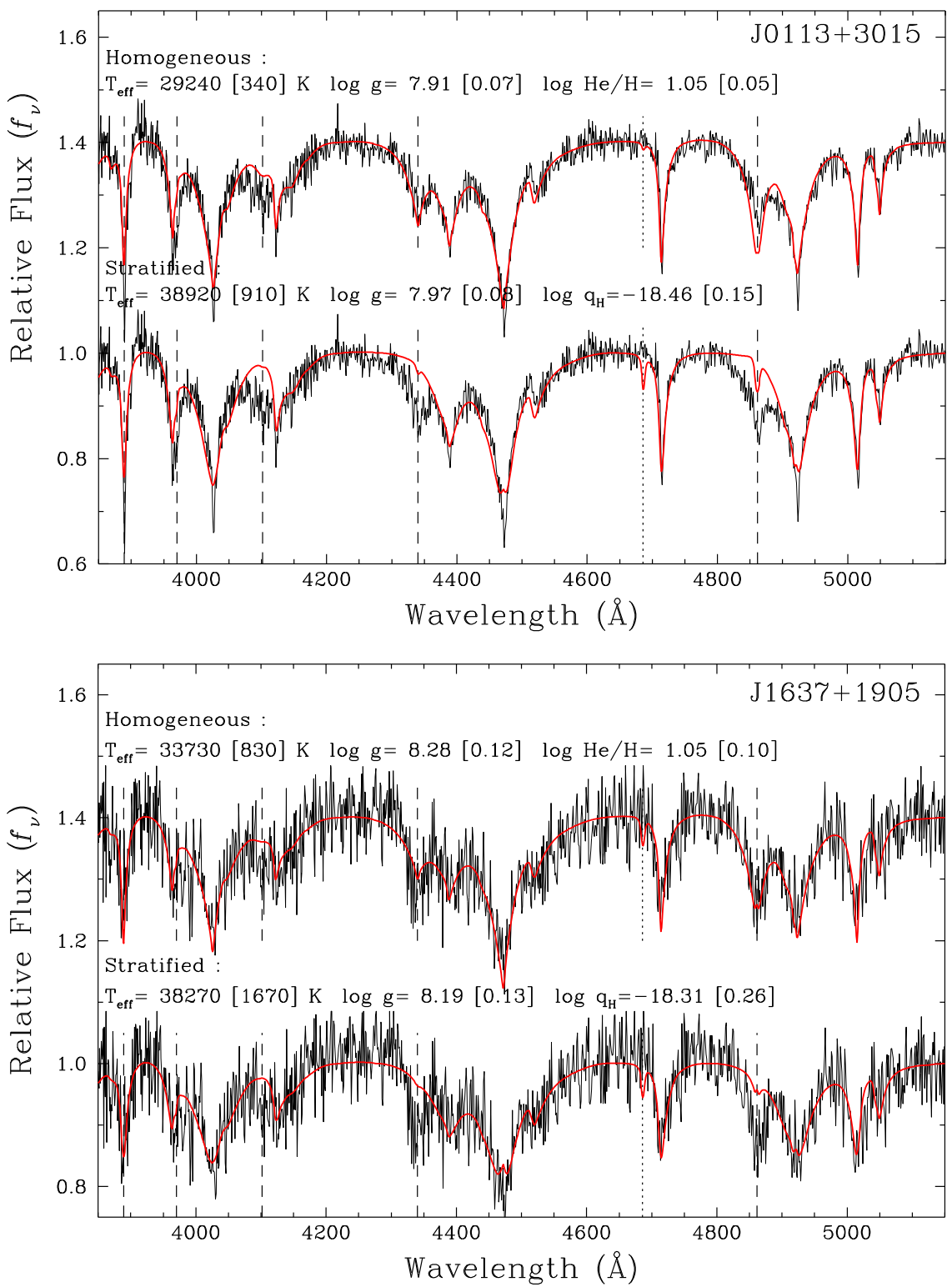

Figure 21. Spectroscopic fits for hot DBA white dwarfs from the SDSS with extreme hydrogen abundances, whose spectra are better fitted with homogeneous models.

homogeneous models-in particular, the He II $\lambda 4686$ absorption feature. Most importantly, the hydrogen abundances inferred in these DBA stars, $N(\mathrm{H}) / N(\mathrm{He}) \sim 0.1$, are in sharp contrast with those reported in normal DBA stars. Bergeron et al. (2011, see their Figure 25), for instance, determined the hydrogen abundances in $\sim 100$ bright DB white dwarfs, and the maximum value measured was around $N(\mathrm{H}) / N(\mathrm{He}) \sim 10^{-3}$ (see also Koester \& Kepler 2015 for a similar result). Furthermore, the hottest white dwarfs in their sample, near $30,000 \mathrm{~K}$, were consistent with having pure helium atmospheres. Lastly, we notice that the DBA star J0113+3015 (Figure 21(a)) has an effective temperature and surface gravity almost identical to those of the DAB J1509-0108 (Figure 14), except that the latter has a stratified atmosphere! Clearly, these two white dwarfs represent key objects in our understanding of the spectral evolution of DB white dwarfs. This is further discussed in the following section.

\section{DISCUSSION}

The results of our spectroscopic analysis are summarized in Table 1, where we separated white dwarfs with stratified atmospheres, cool DOB and DBO stars with spectroscopic solutions consistent with pure helium compositions, and white dwarfs with homogeneous atmospheres. The stars with stratified atmospheres include both those with spectra that are better fitted with stratified models and those for which the stratified solution is simply more sound from an astrophysical point of view. The entries in Table 1 are ordered in each subgroup by right ascension, and we list the values of $T_{\text {eff }}$, $\log g$, the mass derived from the evolutionary models of Wood (1992) with thin hydrogen layers, and either the helium-tohydrogen abundance ratio $(\mathrm{He} / \mathrm{H})$ for homogeneous white dwarfs or the hydrogen layer mass $\left(q_{\mathrm{H}}\right)$ for stratified objects, along with the associated uncertainties (a value of $\log \mathrm{He} / \mathrm{H}=5$ implies a pure helium composition). All objects 
Table 1

Atmospheric Parameters for White Dwarfs

\begin{tabular}{|c|c|c|c|c|c|c|}
\hline Name & Type & $T_{\text {eff }}(\mathrm{K})$ & $\log g$ & $M / M_{\odot}$ & $\log \mathrm{He} / \mathrm{H}$ & $\log q_{\mathrm{H}}$ \\
\hline PG 1305-017 & DAOB & $44,900(500)$ & $7.89(0.04)$ & $0.59(0.02)$ & $\ldots$ & $-16.11(0.07)$ \\
\hline J0033+1422 & DBO & $43,200(600)$ & $8.09(0.09)$ & $0.69(0.05)$ & $\ldots$ & $-17.33(0.19)$ \\
\hline J0342-0722 & $\mathrm{DBO}$ & $48,000(100)$ & $7.86(0.04)$ & $0.58(0.02)$ & $\ldots$ & $-17.78(0.15)$ \\
\hline $\mathrm{J} 0726+3633$ & DBO & $47,500(400)$ & $7.79(0.09)$ & $0.55(0.04)$ & $\ldots$ & $-16.92(0.20)$ \\
\hline $\mathrm{J} 0745+3122$ & DBAO & $40,000(1000)$ & $7.30(0.10)$ & $0.38(0.05)$ & $\ldots$ & $-16.51(0.32)$ \\
\hline $\mathrm{J} 0825+1805$ & DAOB & $50,000(1000)$ & $8.00(0.30)$ & $0.70(0.20)$ & $\ldots$ & $-16.57(0.53)$ \\
\hline J0908+1247 & DAOB & 49,700 (700) & $7.74(0.09)$ & $0.53(0.04)$ & $\ldots$ & $-15.87(0.17)$ \\
\hline J0909+0111 & DOB & $50,600(300)$ & $8.11(0.07)$ & $0.71(0.04)$ & $\ldots$ & $-17.66(0.21)$ \\
\hline $\mathrm{J} 1034+2409$ & $\mathrm{DBO}$ & $49,300(200)$ & $8.00(0.09)$ & $0.64(0.05)$ & $\ldots$ & $-17.60(0.28)$ \\
\hline $\mathrm{J} 1406+5627$ & $\mathrm{DBO}$ & $43,000(2000)$ & $7.90(0.30)$ & $0.60(0.10)$ & $\ldots$ & $-17.12(0.55)$ \\
\hline J1509-0108 & DAB & $29,400(500)$ & $7.90(0.09)$ & $0.56(0.05)$ & $\ldots$ & $-16.78(0.15)$ \\
\hline $\mathrm{J} 1520+2430$ & DAB & $41,000(1000)$ & $7.40(0.10)$ & $0.40(0.05)$ & $\ldots$ & $-15.61(0.26)$ \\
\hline $\mathrm{J} 1548+2031$ & DOAB & $53,200(600)$ & $8.00(0.10)$ & $0.65(0.06)$ & $\ldots$ & $-16.83(0.24)$ \\
\hline $\mathrm{J} 1615+1102$ & DOAB & $52,300(600)$ & $7.90(0.10)$ & $0.61(0.05)$ & $\ldots$ & $-16.63(0.21)$ \\
\hline $\mathrm{J} 2351+0108$ & DAOB & $50,900(600)$ & $7.90(0.10)$ & $0.58(0.05)$ & $\cdots$ & $-16.34(0.22)$ \\
\hline $\mathrm{J} 0005+0038$ & DOB & $50,900(400)$ & $8.02(0.09)$ & $0.66(0.05)$ & $5.00(0.00)$ & $\ldots$ \\
\hline $\mathrm{J} 0341+0053$ & DOB & $51,100(600)$ & $8.00(0.10)$ & $0.63(0.07)$ & $5.00(0.00)$ & $\ldots$ \\
\hline J0531-0008 & DOB & $51,200(900)$ & $7.80(0.20)$ & $0.60(0.10)$ & $2.25(6.12)$ & $\ldots$ \\
\hline $\mathrm{J} 0720+3703$ & DOB & $50,600(600)$ & $7.70(0.10)$ & $0.52(0.04)$ & $5.00(0.00)$ & $\ldots$ \\
\hline $\mathrm{J} 1107+3835$ & DOB & $54,800(900)$ & $7.80(0.10)$ & $0.57(0.06)$ & $0.80(0.20)$ & $\ldots$ \\
\hline $\mathrm{J} 1136+5912$ & DOB & $55,000(2000)$ & $7.70(0.20)$ & $0.52(0.08)$ & $1.05(0.48)$ & $\cdots$ \\
\hline $\mathrm{J} 1259+5723$ & DOB & $52,700(500)$ & $8.10(0.10)$ & $0.68(0.06)$ & $1.52(0.54)$ & $\ldots$ \\
\hline $\mathrm{J} 1509+2922$ & $\mathrm{DBO}$ & $49,800(200)$ & $7.99(0.07)$ & $0.64(0.03)$ & $5.00(0.00)$ & $\ldots$ \\
\hline $\mathrm{J} 1529+1837$ & DOB & $53,000(1000)$ & $8.10(0.20)$ & $0.70(0.10)$ & $1.66(1.62)$ & $\ldots$ \\
\hline $\mathrm{J} 2135+0054$ & DOB & $53,000(2000)$ & $8.00(0.30)$ & $0.70(0.20)$ & $2.16(8.14)$ & $\ldots$ \\
\hline $\mathrm{J} 2156+2047$ & DOB & $51,300(300)$ & $7.89(0.07)$ & $0.60(0.03)$ & $5.00(0.00)$ & $\ldots$ \\
\hline $\mathrm{J} 0113+3015$ & DBA & $29,200(300)$ & $7.91(0.07)$ & $0.57(0.03)$ & $1.05(0.05)$ & $\ldots$ \\
\hline $\mathrm{J} 0816+1316$ & DAO & $56,000(1000)$ & $6.99(0.07)$ & $0.41(0.01)$ & $-1.62(0.11)$ & $\ldots$ \\
\hline $\mathrm{J} 0937+0002$ & $\mathrm{DAO}$ & $46,000(2000)$ & $8.00(0.10)$ & $0.66(0.08)$ & $-1.73(0.20)$ & $\ldots$ \\
\hline $\mathrm{J} 1433+2830$ & DAO & $47,000(2000)$ & $7.10(0.10)$ & $0.37(0.02)$ & $-1.53(0.19)$ & $\ldots$ \\
\hline $\mathrm{J} 1614+3705$ & DAO & $56,000(1000)$ & $7.55(0.09)$ & $0.49(0.03)$ & $-2.63(0.28)$ & $\cdots$ \\
\hline $\mathrm{J} 1637+1905$ & DBA & $33,700(800)$ & $8.30(0.10)$ & $0.79(0.07)$ & $1.05(0.10)$ & $\ldots$ \\
\hline $\mathrm{J} 1704+4141$ & DAO & $41,200(700)$ & $7.05(0.08)$ & $0.36(0.01)$ & $-1.97(0.14)$ & $\ldots$ \\
\hline
\end{tabular}

in this sample are cooler than $T_{\text {eff }}=60,000 \mathrm{~K}$, because this represents the upper limit of our model grid. Our results are also summarized in the $\log g$ versus $T_{\text {eff }}$ diagram displayed in Figure 22, where different symbols indicate the location of the stratified and homogeneous white dwarfs from our analysis, the cool DOB and DBO stars, as well as PG 1305-017.

Overall, we have identified 14 new stratified white dwarfs or stratified candidates in our spectroscopic analysis - a significant improvement over the analysis of Bergeron et al. (1994), who found only one, PG 1305-017. Most stratified white dwarfs in our sample are located in the same region of the $T_{\text {eff }}-\log g$ diagram, that is, with normal surface gravities $(\log g \sim 8)$ and close to the blue edge of the DB gap $\left(T_{\text {eff }} \sim 45,000 \mathrm{~K}\right)$-in the same region as PG 1305-017-with the glaring exception of $\mathrm{J} 0745+3122$ and $\mathrm{J} 1520+2430$, which have significantly lower surface gravities (the two filled squares at the top of Figure 22), and J1509-0108, which lies close to the red edge of the gap instead. We have not found any evidence for stratified white dwarfs within the DB gap (or DB deficiency), although this could be a selection effect in our survey (see below).

The homogeneous white dwarfs in our sample are shown by filled triangles in Figure 22. Particularly interesting here is the location of the 5 DAO stars, also listed in Table 1. For completeness, we also included in this figure the results from Gianninas et al. (2010) for the DAO stars with homogeneous compositions (open triangles), as well as PG 1210+533 (star symbol), a cool DAO star that shows spectroscopic variability (see Figure 22 of Gianninas et al.). Gianninas et al. (and references therein) discuss at length the model where helium in the photosphere of these DAO stars is supported by a weak stellar wind, causing the atmosphere to be chemically homogeneous. For instance, the calculations of Unglaub \& Bues (2000) show that a stellar wind driven by the presence of metals-detected in most DAO stars-can support sufficient amounts of helium in the atmosphere of a white dwarf to explain the observed helium abundances in these objects. There is, however, a limiting temperature for this physical process to operate, known as the "wind limit", below which mass loss ceases, and heavier elements begin to sink under the influence of gravitational settling. Unglaub \& Bues estimate their wind limit for DAO stars at $T_{\text {eff }} \sim 80,000 \mathrm{~K}$, whereas Gianninas et al. (2010) found several DAO stars in the range $60,000<T_{\text {eff }}<80,000 \mathrm{~K}$, and thus suggested an empirical wind limit that extends to much lower effective temperatures, at least down to $T_{\text {eff }}=60,000 \mathrm{~K}$, which is indicated in Figure 22 by the vertical dashed line. One of the DAO stars in our sample, J1614+3705 (Figure 20(d)), falls at the cool end of the Gianninas et al. sample, and in the bulk of DAO stars in terms of $\log g$ values. However, three of our DAO stars have extremely low surface gravities $(\log g \sim 7.0$ - the filled 


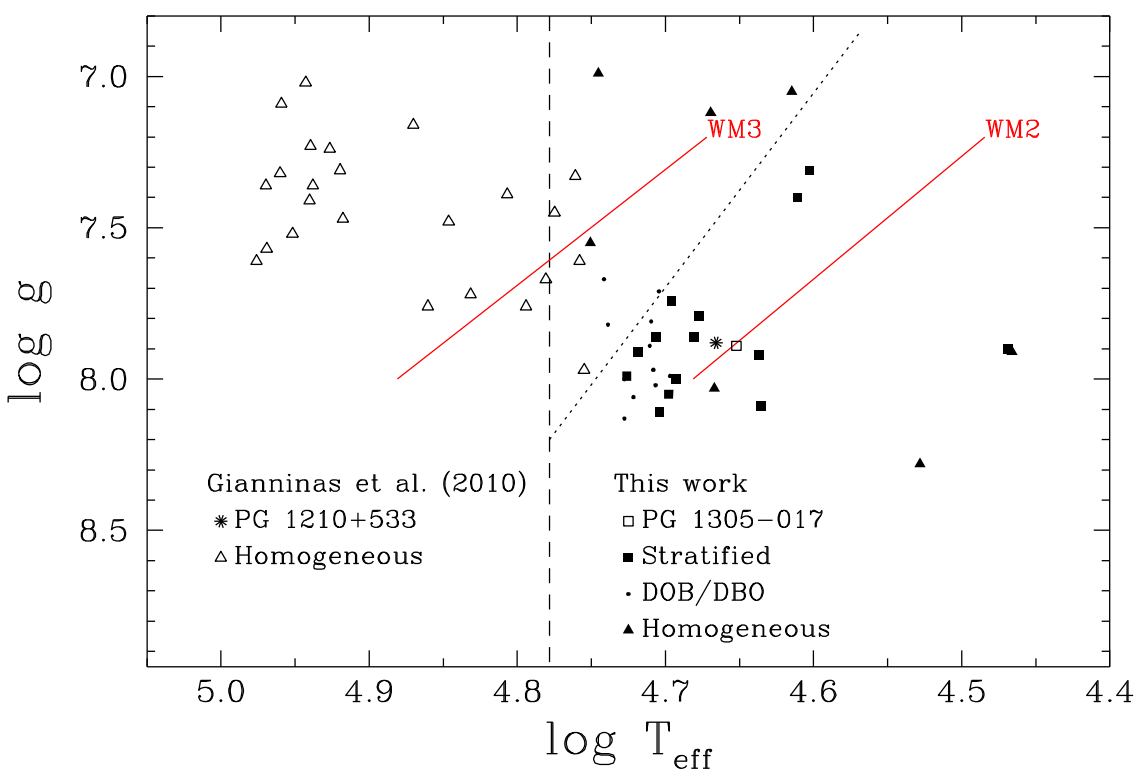

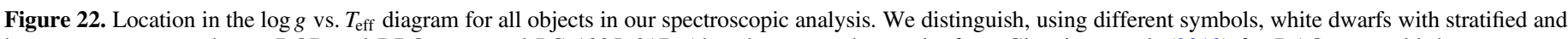

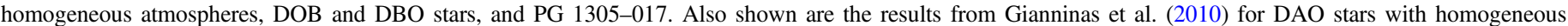

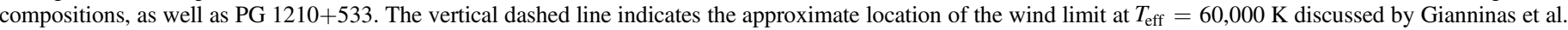

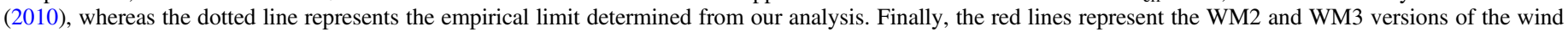
limit determined by Quirion et al. (2012).

triangles at the top of Figure 22), two of which that are found at much lower temperatures. These cool, low surface gravity DAO stars allow us to draw an empirical wind limit shown by the dotted line in this figure. It is, indeed, expected that radiatively supported winds will be more efficient in a low surface gravity white dwarf, as observed here. Interestingly enough, our empirical determination is in excellent agreement with the detailed calculations of Quirion et al. (2012, see their Figure 7) whose so-called WM2 and WM3 versions of their wind limit are reproduced as red lines in Figure 22. These versions correspond to two different mass-loss laws with characteristics detailed in Table 1 and Figure 1 of Quirion et al.

The other homogeneous DAO star in our sample, J0937 +0002 (Figure 20(b)), is much cooler, and close to the blue edge of the DB gap. It is obviously too cool and massive to be explained in terms of a weak stellar wind. Its location in Figure 22 puts it very close to PG $1210+533$, and both objects are actually almost identical twins (see Figure 6 of Bergeron et al. 1994), with He II $\lambda 4686$ observed too deep compared to homogeneous or stratified models. Because PG $1210+533$ has been shown to vary spectroscopically over periods of several years (see Figure 22 of Gianninas et al. 2010), it would be interesting to monitor $\mathrm{J} 0937+0002$ for spectroscopic variability as well. Perhaps these two objects represent the final stage of stratified white dwarfs just about to turn into DA stars, as discussed by Gianninas et al. (2010, see their Section 6.2).

Also shown in Figure 22 is the location of the 11 cool DBO and DOB stars listed in Table 1. As discussed in Section 4.4, despite the values of $\log \mathrm{He} / \mathrm{H}$ reported here, all these white dwarfs are consistent with having pure helium atmospheres. Consequently, their almost perfect overlap in Figure 22 with the stratified white dwarfs identified in our analysis clearly indicates that these stars are not likely to ever turn into DA stars in their lifetime, and that they will always retain a helium-rich atmosphere, an outcome also discussed by MacDonald \& Vennes (1991). These cool DBO/DOB stars represent the obvious progenitors of the hot $\mathrm{DB}$ white dwarfs discovered in the DB gap by Eisenstein et al. (2006b), and most likely the progenitors of most cool DB stars that show no trace of hydrogen whatsoever, reported in the spectroscopic analysis of Bergeron et al. (2011).

Most stratified white dwarfs identified in our survey (with the exception of one) are located, in Figure 22, close to the blue edge of the DB gap — as expected. Indeed, we expect hot white dwarfs with helium-rich atmospheres containing some amount of hydrogen diluted in their stellar envelope to start building a hybrid $\mathrm{H} / \mathrm{He}$ atmosphere when the weak stellar wind dies below the wind limit. These stratified white dwarfs are most likely in the process of turning into DA stars once they cool down to lower temperatures, provided that they still have enough hydrogen in their envelopes continuing to rise to the surface. In some cases, perhaps all the hydrogen has already reached the surface and the star will remain in diffusive equilibrium as a hybrid $\mathrm{H} / \mathrm{He}$ white dwarf while it cools down through the DB gap. There is no easy way to tell. We believe J1509-0108 (Figure 14) may represent such an object still in diffusive equilibrium. After all, the hydrogen layer mass for this object, $\log q_{\mathrm{H}}=-16.7$, is not particularly thin or thick with respect to other stratified white dwarfs in our sample. Thus, it is reasonable to assume that all the hydrogen in this DAB star at $T_{\text {eff }} \sim 30,000 \mathrm{~K}$ has had plenty of time to diffuse to the surface. As it cools further, the helium convection zone will become efficient enough to turn this stratified object into a chemically homogeneous DBA white dwarf such as J0113 +3015 (Figure 21(a)), with an unusually large hydrogen-tohelium abundance ratio. If this interpretation is correct, cooler DBA stars, which are usually interpreted as the DA to DB transformation resulting from the convective dilution of a thin hydrogen atmosphere with the underlying convective helium envelope (see Bergeron et al. 2011 and references therein), may have a different origin - or at least, a fraction of them. Indeed some could have simply evolved from chemically stratified hybrid $\mathrm{H} / \mathrm{He}$ white dwarfs directly into DBA stars, without going through a DA phase. This could perhaps explain the 
origin of the three most hydrogen-rich DBA stars near 25,000 K observed in Figure 25 of Bergeron et al. (2011).

As discussed above, we have not found any stratified white dwarfs between $T_{\text {eff }} \sim 30,000$ and $40,000 \mathrm{~K}$, but this is probably a selection effect due to the fact that our search was aimed at much hotter white dwarfs with He II $\lambda 4686$ visible. Thus, it is very likely that more chemically stratified white dwarfs will be identified in the spectroscopic analysis of DB stars in the SDSS, such as that of Koester \& Kepler (2015).

The spectral evolution of white dwarfs with traces of hydrogen in their envelope, which eventually appear as hybrid spectra, is a most complicated research topic, as discussed at length by MacDonald \& Vennes (1991). The SDSS offers a unique opportunity to study the various physical processes at work by significantly increasing the sample of known hybrid white dwarfs. A study such as ours represents another step in our global understanding of the spectral evolution of white dwarf stars.

We thank A. Gianninas for a careful reading of our manuscript. We would also like to thank the Director and staff of Steward Observatory for the use of their facilities, as well as the Director and staff of Gemini South Observatories for the remote observing. This work was supported in part by the NSERC Canada and by the Fund FRQ-NT (Québec). Based on observations obtained at the Gemini Observatory (program GS2015B-Q-68), which is operated by the Association of Universities for Research in Astronomy, Inc., under a cooperative agreement with the NSF on behalf of the Gemini partnership: the National Science Foundation (United States), the National Research Council (Canada), CONICYT (Chile), Ministerio de Ciencia, Tecnología e Innovación Productiva (Argentina), and Ministério da Ciência, Tecnologia e Inovação (Brazil).

\section{REFERENCES}

Bergeron, P., Saffer, R., \& Liebert, J. 1992, ApJ, 394, 228

Bergeron, P., Saumon, D., \& Wesemael, F. 1995, ApJ, 443, 764

Bergeron, P., Wesemael, F., Beauchamp, A., et al. 1994, ApJ, 432, 305

Bergeron, P., Wesemael, F., Dufour, P., et al. 2011, ApJ, 737, 28

Dumont, S., \& Heidmann, N. 1973, A\&A, 27, 273

Eisenstein, D. J., Liebert, J., Harris, H. C., et al. 2006a, ApJS, 167, 40

Eisenstein, D. J., Liebert, J., Koester, D., et al. 2006b, AJ, 132, 676

Fontaine, G., Brassard, P., \& Bergeron, P. 2001, PASP, 113, 409

Fontaine, G., \& Wesemael, F. 1987, in IAU Colloq. 95, The Second Conference on Faint Blue Stars, ed. A. G. D. Philip, D. S. Hayes, \& J. Liebert (Schenectady, NY: Davis), 319

Gianninas, A., Bergeron, P., Dupuis, J., \& Ruiz, M. T. 2010, ApJ, 720, 581

Green, R., Schmidt, M., \& Liebert, J. 1986, ApJS, 61, 305

Hügelmeyer, S. D., Dreizler, S., Homeier, D., et al. 2006, A\&A, 454, 617

Jordan, S., \& Koester, D. 1986, A\&AS, 65, 367

Kepler, S. O., Pelisoli, I., Koester, D., et al. 2015, MNRAS, 446, 4078

Kepler, S. O., Pelisoli, I., Koester, D., et al. 2016, MNRAS, 455, 3413

Kleinman, S. J., Harris, H. C., Eisenstein, D. J., et al. 2004, ApJ, 607, 426

Kleinman, S. J., Kepler, S. O., Koester, D., et al. 2013, ApJS, 204, 5

Koester, D., \& Kepler, S. O. 2015, A\&A, 583, A86

MacDonald, J., \& Vennes, S. 1991, ApJ, 371, 719

McCook, G. P., \& Sion, E. M. 1999, ApJS, 121, 1

Press, W. H., Flannery, B. P., \& Teukolsky, S. A. 1986, Numerical recipes: The Art of Scientific Computing (Cambridge: Cambridge Univ. Press)

Quirion, P.-O., Fontaine, G., \& Brassard, P. 2012, ApJ, 755, 128

Savitzky, A., \& Golay, M. J. E. 1964, AnaCh, 36, 1627

Sion, E. M., Greenstein, J. L., Landstreet, J. D., et al. 1983, ApJ, 269, 253

Tremblay, P.-E., \& Bergeron, P. 2009, ApJ, 696, 1755

Tremblay, P.-E., Ludwig, H.-G., Freytag, B., et al. 2015, ApJ, 799, 142

Tremblay, P.-E., Ludwig, H.-G., Steffen, M., \& Freytag, B. 2013, A\&A, 559, A104

Unglaub, K., \& Bues, I. 2000, A\&A, 359, 1042

Vennes, S., \& Fontaine, G. 1992, ApJ, 401, 288

Werner, K. 1996, ApJL, 457, L39

Wood, M. A. 1992, ApJ, 386, 539 